\title{
Identification of critical mechanical parameters for ad- vanced analysis of masonry arch bridges
}

\author{
Enrico Tubaldi ${ }^{\mathrm{a}, \mathrm{b} *}$, Lorenzo Macorini ${ }^{\mathrm{a}}$, Bassam A. Izzuddin ${ }^{\mathrm{a}}$ \\ ${ }^{a}$ Department of Civil and Environmental Engineering, Imperial College, London, UK; ${ }^{b}$ De- \\ partment of Civil and Environmental Engineering, University of Strathclyde, Glasgow, UK; \\ *enrico.tubaldi@strath.ac.uk
}

\begin{abstract}
The response up to collapse of masonry arch bridges is very complex and affected by many uncertainties. In general, accurate response predictions can be achieved using sophisticated numerical descriptions, requiring a significant number of parameters that need to be properly characterised. This study focuses on the sensitivity of the behaviour of masonry arch bridges with respect to a wide range of mechanical parameters considered within a detailed modelling approach. The aim is to investigate the effect of constitutive parameters variations on the stiffness and ultimate load capacity under vertical loading. First, advanced numerical models of masonry arches and of a masonry arch bridge are developed, where a mesoscale approach describes the actual texture of masonry. Subsequently, a surrogate kriging metamodel is constructed to replace the accurate but computationally expensive numerical descriptions, and global sensitivity analysis is performed to identify the mechanical parameters affecting the most the stiffness and load capacity. Uncertainty propagation is then performed on the surrogate models to estimate the probabilistic distribution of the response parameters of interest. The results provide useful information for risk assessment and management purposes, and shed light on the parameters that control the bridge behaviour and require an accurate characterisation in terms of uncertainty.
\end{abstract}

Keywords: Global sensitivity analysis; masonry arch bridges; Kriging; local sensitivity analysis; uncertainty propagation; random variables. 


\section{Introduction}

Masonry arch bridges are very durable structures, which still constitute a significant proportion of the bridge stock in Europe. Their importance for the road and rail infrastructure network is also demonstrated by the considerable amount of experimental investigations and numerical studies devoted to the safety assessment of masonry arch bridges (Orban, 2007; Melbourne et al., 2007; Sarhosis et al., 2017). The effects of ageing, the increase in time of traffic loading and of other type of hazards (e.g., floods) require a continuous development and improvement of procedures for their evaluation, monitoring, and management. This is of paramount importance to ensure that these structures can continue to operate in the future.

The behaviour of masonry arch bridges is very complex, strongly nonlinear and dependent on the properties of the many and interconnected constituent components of the system, such as the masonry arch barrel and piers, the backfill, and the spandrel walls. Thus, an accurate simulation of their behaviour and interaction is essential for predicting the bridge response up to collapse. A preliminary assessment can be carried out using semi-empirical formulas (Page, 1993) or limit analysis approaches (Gilbert, 2007, Zampieri et al., 2015) such as those based on Heyman's theory (Heyman, 1969). However, a realistic evaluation of the behaviour of masonry arch bridges under both service and extreme loadings requires resorting to three-dimensional non-linear models, such as discrete element models or those employing a mesoscale description of masonry (Sarhosis et al., 2017; Zhang et al., 2018). These approaches generally have a particularly high computational cost, and are not suitable for application in common practice but rather for calibrating and validating simplified modelling approaches.

Besides the complexity in simulating the structural behaviour, one important aspect that should be addressed in any assessment of masonry arch bridges concerns the many uncertainties related to the geometrical and material parameters of the bridge components (Brencich et al, 2017; Casas, 2011; De Felice \& De Santis, 2010; Cavalagli et al., 2017; Ng and Fairfield, 2002; 
Moreira et al., 2016; Moreira et al., 2017; Hacıefendioğlu et al., 2017). In general, the values of these parameters are usually unknown and characterised by a significant scatter. For example, the constitutive behaviour of masonry components is strongly affected by the quality of workmanship and weather conditions. Even in the case of newly built masonry structures, when bricklaying is carried out by the same bricklayer, it is difficult to obtain brickwork with consistent mechanical properties (Jie et al., 2017; Melbourne \& Gilbert, 1995; Tabbakhha \& Modaressi-Farahmand-Razavi, 2016; Tabbakhha \& Deodatis, 2017; Schueremans \& Van Gemert, 2006). The uncertainty and scatter in masonry material properties is even higher in the case of existing masonry arch bridges, which were often built using material available in place with scarce quality control, and subjected to ageing, material deterioration, defects, and environmental effects (Zanaz et al.; 2016; Sykora and Holicky, 2016). A large number of tests are usually needed to obtain statistically meaningful values for the masonry properties (Schueremans \& Van Gemert, 2006; Sykora and Holicky, 2016). However, it is often difficult to extract specimens to be tested in laboratory, and, in the case of historical buildings, highly invasive testing is not possible at all. To overcome this issue, the use of inverse analysis procedures has been proposed to estimate the main parameters of masonry models based on flat-jack tests (Chisari et al., 2018). Also, the material employed for the backfill is usually characterised by significant uncertainty (Ng \& Fairfield, 2002; Andersson, 2011).

The works of Zhang et al. (2018) and of Moreira et al. (2016) and Moreira et al. (2017) have shown that even small variations of the parameters characterising the masonry and the fill material may result in significant changes of the bridge collapse load. This issue, together with the high uncertainty of bridge material parameters, suggests that deterministic analysis approaches are inadequate for evaluating the safety of masonry arch bridges and that the use of probabilistic methodologies should be considered. In this respect, there are many methodologies available 
for uncertainty propagation and uncertainty quantification. Monte Carlo simulation is the reference one, the easiest to implement, but also very computational expensive, whereas variance reduction techniques, such as Latin Hypercube Sampling and Subset simulation (Au and Wang, 2014), enable a significant reduction of the number of simulations required by uncertainty propagation. However, their use remains impractical when dealing with complex FE model and time-consuming analyses. For this reason, metamodels such as response surface (Myers \& Montgomery, 1998) and kriging (Sacks et al., 1989) have gained popularity over the past few decades. These tools permit to replace the computational expensive FE model for the purpose of mapping the input (uncertain parameters) and the output (response), with some applications also in the context of assessment of masonry structures (Chisari et al., 2018; Giannini et al., 1996; Schueremans and Gemert, 1999; Milani \& Benasciutti, 2010).

In order to carry out a probabilistic analysis of masonry arch bridges, it is necessary to know the statistical distribution of the uncertain bridge parameters. However, the available information is often very scarce (Melbourne et al., 2007), and a relatively high number of tests must be carried out to retrieve the material properties of a composite material such as masonry (Schueremans et al., 2006). This problem could limit significantly the use of advanced FE model such as those based on a mesoscale or micro-scale description of masonry, given the large number of input parameters required for their definition. Thus, before carrying any characterisation tests and probabilistic analysis of a bridge it would be useful to identify the parameters that control the bridge response. Sensitivity analysis toolboxes such as Local Sensitivity Analysis (LSA) and Global Sensitivity Analysis (GSA) (Patelli et al., 2010; Saltelli \& Chan, 2000; Sobol, 1993) can be used for distinguishing the model parameters that affect the most the response parameters of interest and those that can be assumed as fixed. More in general, the 
results of sensitivity analysis can be very useful for input variables' screening, model calibration, model validation, and decision-making processes (Patelli et al., 2010; Saltelli \& Chan, 2000; Queipo et al., 2005; Rohmer \& Foerster, 2011).

In Mukherjee et al. (2011), Sobol's GSA (Sobol, 1993) was employed to investigate the parameters that affect the strength of a mesoscale model of a masonry wall. It was found that the friction coefficient of mortar joints is the most sensitive one, followed by Young's modulus of mortar, thickness of the mortar joint, compressive fracture energy and compressive strength of the masonry. Zhu et al. (2017) employed Sobol's method for GSA to evaluate the parameters controlling the compressive strength of a micro-scale model of a masonry wallet, and found that the tensile strength of the block is the most important one, followed by the elastic modulus of the block, Poisson's ratio of mortar, and elastic modulus of the mortar.

Some results of application of sensitivity analysis to masonry arch bridges are already available. For example, in Moreira et al. (2016) and in Moreira et al. (2017), sensitivity analysis was employed to evaluate the most influential parameters within a probabilistic framework for collapse assessment of masonry arch bridges through limit analysis. However, Sobol's GSA has yet to be employed to evaluate the most critical parameters of advanced meso-scale models of masonry arch bridges. Thus, this paper has two objectives: i) identify, via Sobol's GSA, the mechanical parameters with the highest influence on the stiffness and capacity of arches and masonry arch bridges, and ii) analyse how the uncertainty in these parameters propagates to the response of bridges and to the collapse probability. For this purpose, different benchmark examples are considered. The first one is based on a widely employed analytical expression for computing the compressive strength of the mortar and of the bricks. This preliminary example, already considered in a similar study on the use of surrogate models for probabilistic analysis of masonry structures (Milani \& Benasciutti, 2010), is used to test the validity and accuracy of 
the kriging metamodels for sensitivity and uncertainty propagation studies. The other two examples consist of numerical models of single arches with different rise-to-span ratio, and of a full masonry arch bridge. An advanced mesoscale modelling strategy developed at Imperial College (Macorini \& Izzuddin, 2011) and already validated based on comparison with experimental tests is employed to describe the structural behaviour of these structures. In order to alleviate the computational cost of the analyses, a metamodel is proposed in this work based on the results obtained by running few structural finite element analyses for different combinations of the input parameters, and then used as an inexpensive mathematical approximation of the actual FE model. In particular, the metamodel is employed for carrying out the global sensitivity analysis and for propagating the uncertainty in the input parameters and estimating the output probability distribution of interest.

\section{Methodology}

This section illustrates the approach and tools employed for finding the mechanical parameters that influence the most the capacity of arches and masonry arch bridges, and for the uncertainty propagation. Firstly, the proposed FE modelling strategy based on a mesoscale description of the masonry arch barrel is briefly recalled in Section 2.1. Subsequently, the basic concept of kriging surrogate, which is used for sensitivity analysis and uncertain propagation, is described together with his construction based on a reduced number of FE analyses. Finally, Section 2.3 illustrates the methods employed for local and global sensitivity analysis.

\subsection{Finite element modelling approach}

In the following, the approach developed at Imperial College for describing masonry arch bridges utilising a mesoscale strategy for brick/block-masonry (Macorini \& Izzuddin, 2011) is briefly illustrated. The approach, implemented in ADAPTIC (Izzuddin, 1991), was initially developed for simulating the behaviour of masonry arches by accounting for the actual masonry 
bond (Zhang et al., 2016; Zhang et al., 2018b). It was later extended to allow for the simulation of the backfill and spandrel walls contribution and their interaction with the arch barrel (Tubaldi et al., 2018a; Minga et al., 2018).

The mesoscale approach is enhanced using partitioned modelling for parallel processing on high performance computing (Jokhio \& Izzuddin, 2015) (Figure 1), where the masonry arch is described using 20-noded 3D elastic solid elements to model masonry units and 16-noded 2D nonlinear zero thickness interface elements to account for the formation of cracks in correspondence of the mortar joints.

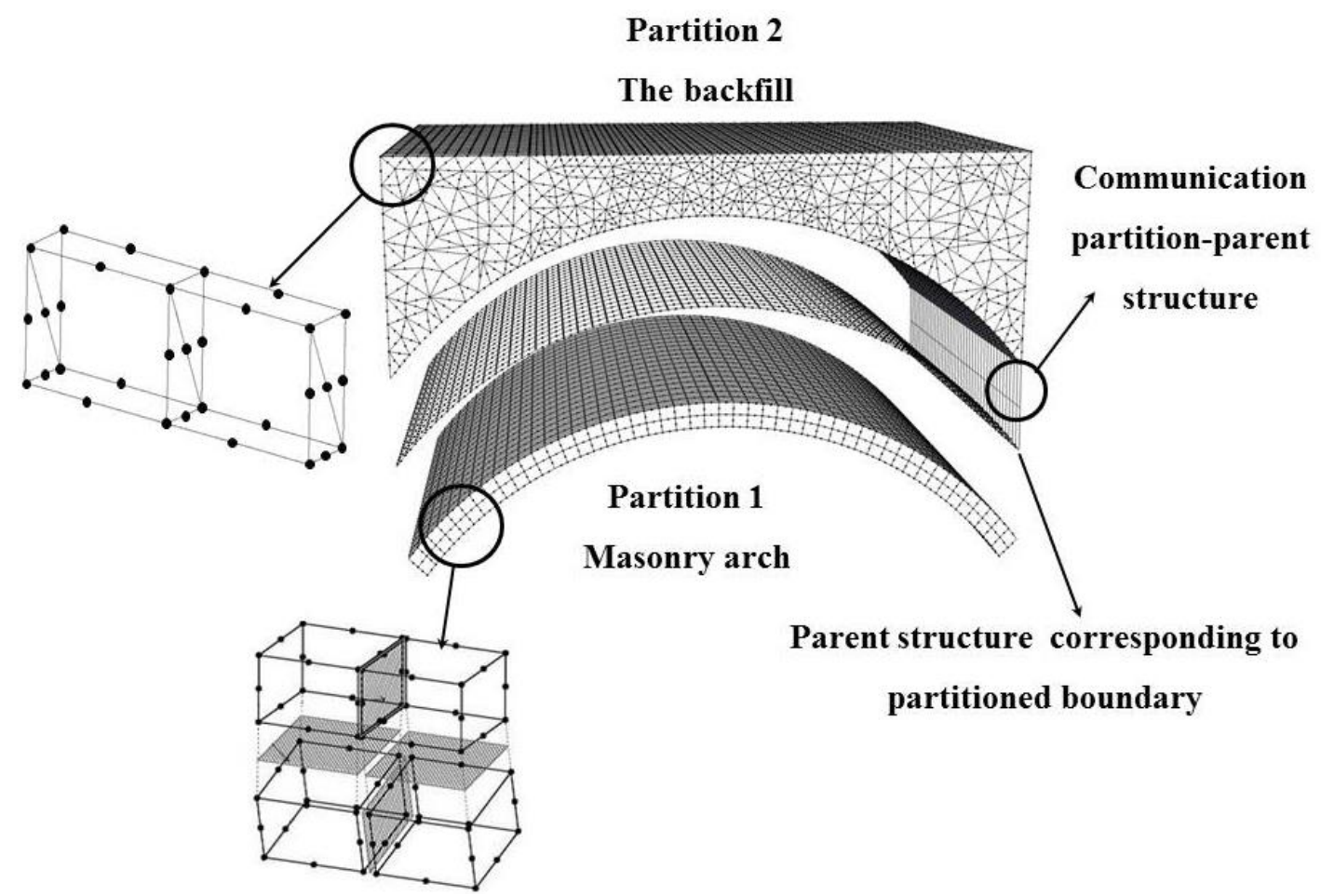

Figure 1. Mesoscale partitioned modelling approach for masonry arch bridges (after Zhang et al. (2018a)).

Additional nonlinear interface elements can be placed also in the middle of each bricks to capture potential development of cracks, though this is not necessary for the arches and bridges analysed in this study as efficient strip models (Zhang et al. 2016, Zhang et al. 2018b) are employed to investigate the critical mechanical parameters of masonry arches, and the arches are characterised by stretcher bonding. In these cases, the response up to collapse is characterised mainly by the development of radial and circumferential cracks along mortar joints (Zhang 
et al. 2016, Zhang et al. 2018b), which does not require the representation of cracking in masonry units".Geometric nonlinearity is described by using a corotational formulation whereas material nonlinearity is accounted for employing a robust coupled damaged-plasticity cohesive model (Minga et al., 2018) enabling an effective representation of damage, cracks and plastic separations (Figure 2).

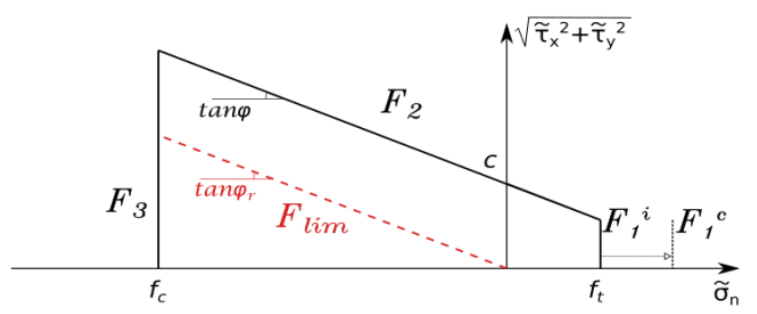

Figure 2. Multi-surface yield criterion and evolution of nonlinear interface elements (after Minga et al. (2018)).

With regards to the backfill, a realistic representation of its behaviour and of the interaction with the arch barrel is essential for an accurate response prediction of masonry arch bridges. In the proposed modelling strategy, the backfill domain is discretised using 15-noded tetrahedral elements with an elasto-plastic material behaviour. The isotropic elastic response is described adopting specific values for the Young's modulus and Poisson's ratio, whereas the plastic behaviour is modelled by employing a modified Drucker-Prager (D-P) yield criterion (Figure 3), with tension and compressive caps as described in (Tubaldi et al., 2018). The spandrel walls can also be described in a simplified way with a similar model, by adding also damage to account for the brittle behaviour of masonry (Tubaldi et al., 2018).

a)

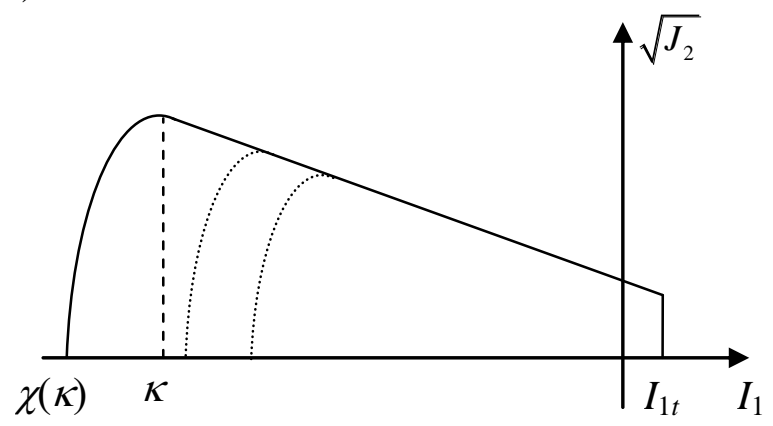

b)

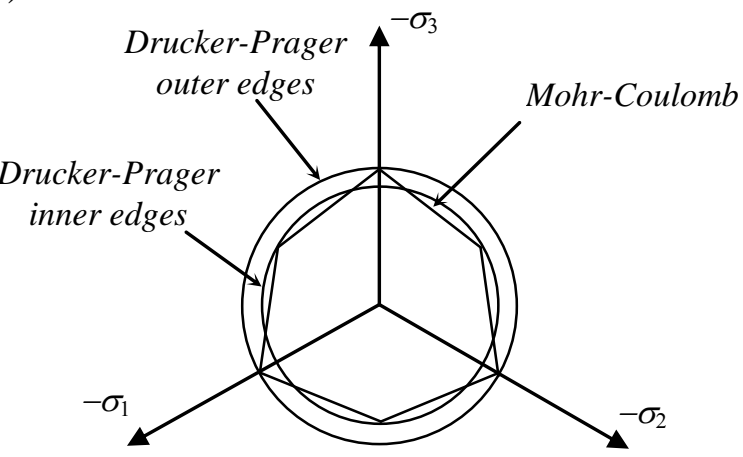


Figure 3. a) Modified D-P model with tensile cap and elliptic cap in compression, b) fitting of the D-P yield surface to the M-C yield surface in the octahedral plane

A mortar mesh tying method for non-conforming interfaces (Minga et al., 2018) is employed to allow the backfill and the arch barrel domains to be meshed independently without compatibility considerations, thus enabling the optimisation of the individual meshes. In particular, a coarser mesh can be employed at the arch barrel-backfill interface, without compromising the accuracy of the results. The interaction between the arch and the backfill is described by employing nonlinear interface elements to represent separation and frictional sliding at the archbackfill physical interface. These interface elements connect the set of nodes at the extrados of the arch barrel with a set of coincident nodes, which are then tied to the nodes at the intrados of the backfill.

Finally, a hierarchical partitioned modelling strategy (Jokhio \& Izzuddin, 2015; Jokhio \& Izzuddin, 2013; Jokhio, 2012) allowing for parallel computations and embedded in ADAPTIC (Izzuddin, 1991), is employed to reduce the time of the analysis. This is particularly beneficial for large-scale FE models but also for the problem at hand, requiring a significant number of simulations.

The proposed finite element modelling strategy has been validated by comparison with available experimental tests on a wide range of masonry structures. In particular, it can simulate with good accuracy the behaviour up to collapse of masonry arches (Zhang et al., 2016; Zhang et al., 2018b) and masonry arch bridges (Zhang et al., 2018a; Tubaldi et al., 2018). It is noteworthy that in the case of square arch bridges subjected to line loads uniformly distributed along the bridge width, an efficient representation of the behaviour can obtained by employing strip models, with only one set of solid elements along the width of the bridge specimen for the arch and the backfill meshes (Zhang et al. 2018a). Obviously, this representation is accurate in the case of rigid spandrel walls detached from the arch barrel but providing transverse confinement to 
the backfill, whereas it provides only a crude description of the bridge behaviour in the case of short widths and spandrel walls attached to the arch.

\subsection{Kriging surrogate}

Let $f: R^{n_{x}} \rightarrow R$ formalise the relation $y=f(\mathbf{x})$, evaluated by means of FE calculations, between a scalar output $y \in R$ (e.g., the bridge collapse load) and a set of input variables collected in the $n_{x}$ dimensional vector $\mathbf{x}=\left[\begin{array}{lll}x_{1} & \ldots & x_{n_{x}}\end{array}\right]^{T}$, where $\mathbf{x} \in R^{n_{x}}$. A Kriging metamodel provides an inexpensive mathematical approximation of the actual, yet time-consuming FE simulation. The construction of the kriging metamodel relies on sampling the input space at $n_{s}$ unique locations to obtain response values for the output through the FE model. The sampling process is also known as design of experiments (Queipo et al. 2005), and the $n_{s}$ samples of the input and of the corresponding response are called support points or training set (Jia and Taflanidis, 2013). The design of experiments can be carried out by employing Latin Hypercube sampling or via any other technique such as Sobol sampling (Sobol', 1976), which permit to cover the expected range of variation of the components of $\mathbf{x}$. This obviously requires knowledge of the range in which the kriging metamodel will be used so that the support points extend over this range. It is noteworthy that the FE models addressed here are deterministic, and thus repeated runs for the same input parameters give the same response.

The idea behind Kriging metamodelling is to consider the output as a realisation of a stochastic process:

$$
y(\mathbf{x})=\mu(\mathbf{x})+Z(\mathbf{x})
$$

where $\mu(\mathbf{x})$ is a deterministic function approximating the mean (global) trend of the output, while $Z(\mathbf{x})$ creates a "localised" departure from it. This departure is assumed to be a Gaussian process with zero mean, constant variance $\sigma^{2}$, and stationary autocorrelation function $R\left(\mathbf{x}, \mathbf{x}^{\prime}\right)$ 
such that the autocovariance function is $\operatorname{Cov}\left[Z(\mathbf{x}) Z\left(\mathbf{x}^{\prime}\right)\right]=\sigma^{2} R\left(\mathbf{x}, \mathbf{x}^{\prime}\right)$. In universal kriging, the trend is expressed as a linear combination of simple regression functions:

$$
y(\mathbf{x})=\mathbf{f}(\mathbf{x})^{T} \boldsymbol{\alpha}+Z(\mathbf{x})
$$

where $\mathbf{f}(\mathbf{x})=\left[f_{1}(\mathbf{x}) \ldots f_{p}(\mathbf{x})\right]^{T}$ is the basis function vector, $p$ is the total number of basis functions, and $\boldsymbol{\alpha}=\left[\alpha_{1} \ldots \alpha_{p}\right]^{T}$ is the unknown coefficients vector.

For the autocorrelation function, a popular choice is the anisotropic Gaussian correlation (Jia and Taflanidis, 2013):

$$
R\left(\mathbf{x}^{l}, \mathbf{x}^{m}\right)=\prod_{i=1}^{n_{x}} \exp \left[-\theta_{i}\left|x_{i}^{l}-x_{i}^{m}\right|^{2}\right]
$$

where a univariate correlation with weight $\theta_{i}$ is used for the $i$-th of the $n_{x}$ dimensions of $\mathbf{x}$, leading to a weight vector $\boldsymbol{\theta}=\left[\theta_{i} \ldots \theta_{n_{x}}\right]$.

For the set of $n_{s}$ observations with input matrix $\mathbf{X}=\left[\mathbf{x}^{1} \ldots \mathbf{x}^{n_{s}}\right]$, such that $\mathbf{X} \in \square^{n_{x} \times n_{s}}$, and corresponding output vector $\mathbf{Y}=\left[y\left(\mathbf{x}^{1}\right) \ldots y\left(\mathbf{x}^{n_{S}}\right)\right]^{T}$, with $\mathbf{Y} \in \square^{n_{S} \times 1}$, the $\mathbf{F}(\mathbf{x}) \in \square^{n_{s} \times p}$ basis matrix $\mathbf{F}(\mathbf{x})=\left[\mathbf{f}\left(\mathbf{x}^{1}\right) \ldots \mathbf{f}\left(\mathbf{x}^{n_{S}}\right)\right]^{T}$ is defined, together with the $\mathbf{R} \in \square^{n_{S} \times n_{S}}$ correlation matrix $\mathbf{R}$ , whose $l m$-th element is $R\left(\mathbf{x}^{l}, \mathbf{x}^{m}\right)$, for $l, m=1,2, \ldots, n_{s}$. Also, for the new input $\mathbf{x}$ at which the response needs to be estimated, the correlation vector $r(\mathbf{x})=\left[R\left(\mathbf{x}, \mathbf{x}^{1}\right) \ldots R\left(\mathbf{x}, \mathbf{x}^{n_{s}}\right)\right]^{T}$ between $\mathbf{x}$ and the observation points of $\mathbf{X}$ is defined, with $r(\mathbf{x}) \in \square^{n_{s} \times 1}$.

A linear kriging predictor is employed, where the estimate of the response at $\mathbf{x}$ is expressed based on the response values $\mathbf{Y}$ as $\hat{y}(\mathbf{x})=\mathbf{c}^{T} \mathbf{Y}$. The best linear unbiased kriging predictor cor- 
responds to the value of $\mathbf{c}$ minimising the mean square error $\phi(\mathbf{x})=E\left[(\hat{y}(\mathbf{x})-y(\mathbf{x}))^{2}\right]$ subject to the unbiasedness constraint $E[\hat{y}(\mathbf{x})-y(\mathbf{x})]=0$, where $E[\cdot]$ denotes the expected value operator.

This leads to the following estimator:

$$
\hat{y}(\mathbf{x})=\mathbf{f}(\mathbf{x})^{T} \boldsymbol{\alpha}^{*}+r(\mathbf{x})^{T} \boldsymbol{\beta}^{*}
$$

where:

$$
\boldsymbol{\alpha}^{*}=\left(\mathbf{F}^{T} \mathbf{R}^{-1} \mathbf{F}\right)^{-1} \mathbf{F}^{T} \mathbf{R}^{-1} \mathbf{Y}, \quad \boldsymbol{\beta}^{*}=\mathbf{R}^{-1}\left(\mathbf{Y}-\mathbf{F} \boldsymbol{\alpha}^{*}\right)
$$

with $\boldsymbol{\alpha}^{*} \in \square^{p \times 1}$ and $\boldsymbol{\beta}^{*} \in \square^{n_{s} \times 1}$. The second term of Equation (4), representing the localised deviation from the global trend, is such that it weights more the points in the training set $\mathbf{X}$ that are closer to the target point $\mathbf{x}$.

The weighting factors $\boldsymbol{\theta}=\left[\theta_{i} \ldots \theta_{n_{x}}\right]$, together with the process variance $\sigma^{2}$, can be derived by using the Maximum Likelihood Estimation (MLE) method, i.e. by maximising the probability of the $n_{s}$ observations given the weights $\boldsymbol{\theta}$ and $\sigma^{2}$ (Lophaven et al., 2002).

Ultimately, the kriging predictor yields an estimate of $y(\mathbf{x})$ that is a Gaussian random variable with mean $\hat{y}(\mathbf{x})$ and variance $\hat{\phi}(\mathbf{x})$ given by:

$$
\begin{aligned}
& \hat{\phi}^{2}(\mathbf{x})=\sigma^{2}(\mathbf{x})\left[1+\mathbf{u}^{T}\left(\mathbf{F}^{T} \mathbf{R}^{-1} \mathbf{F}\right)^{-1} \mathbf{u}-\mathbf{r}(\mathbf{x})^{T} \mathbf{R}^{-1} \mathbf{r}(\mathbf{x})\right] \\
& \mathbf{u}=\mathbf{F}^{T} \mathbf{R}^{-1} \mathbf{r}(\mathbf{x})-\mathbf{f}(\mathbf{x})
\end{aligned}
$$

A Kriging metamodel has many interesting features (Jia and Taflanidis, 2013). First, it is an exact interpolator, i.e., the prediction is exact at the training points and the associated variance is zero. Second, it is asymptotically consistent (provided that the auto-correlation function is regular), i.e. increasing the number of observations decreases the overall variance of the process. 
Last, the prediction at a given point $\mathbf{x}$ is considered as a realisation of a Gaussian random variable, for which it is possible to derive confidence bounds on a prediction.

The global accuracy of the metamodel can be described by carrying a leave-one-out crossvalidation and by evaluating the coefficient of determination (Rohmer \& Foerster, 2011):

$$
Q^{2}=1-\frac{\sum_{i=1}^{n_{s}}\left(y\left(\mathbf{x}_{i}\right)-\hat{y}^{(-i)}\left(\mathbf{x}_{i}\right)\right)^{2}}{\frac{1}{N} \sum_{i=1}^{N}\left(y\left(\mathbf{x}_{i}\right)-\bar{y}\right)^{2}}
$$

where $y\left(\mathbf{x}_{i}\right)$ is the actual response value at $\mathbf{x}_{i}$ (training point), $\bar{y}$ is the sample mean, and $\hat{y}^{(-i)}\left(\mathbf{x}_{i}\right)$ is the response prediction obtained by fitting a metamodel on all the training samples except $\mathbf{x}_{i}$. Values of $R^{2}$ higher than $80 \%$ correspond to a satisfactory quality of the metamodel (Rohmer \& Foerster, 2011).

The proposed metamodel can be employed to replace the original FE model for the purpose of carrying out GSA but also for uncertainty propagation in the case of random inputs with joint probability density function $p_{\mathbf{x}}(\mathbf{x})$.

\subsection{Local and global sensitivity analysis}

In general, sensitivity analysis aims to investigate how changes in the model input $\mathbf{x}$ influence the output $y$ (Saltelli and Chan, 2000; Queipo et al., 2005). LSA studies the effect of small input perturbations of $\mathrm{x}$ around a nominal or central value, denoted as $\mathrm{x}_{0}$. The first-order truncated Taylor series expansion of $y(\mathbf{x})$ is used to approximate the input-output relationship in the neighborhood of $\mathbf{x}_{0}$ :

$$
y(\mathbf{x})=y\left(\mathbf{x}_{0}\right)+\nabla y\left(\mathbf{x}-\mathbf{x}_{0}\right)
$$

where $\nabla y=\left[\begin{array}{lll}\left.\frac{\partial y}{\partial x_{1}}\right|_{\mathbf{x}_{0}} & \ldots & \left.\frac{\partial y}{\partial x_{k}}\right|_{\mathbf{x}_{0}}\end{array}\right]$ is the local gradient of the output at $\mathbf{x}_{0}$. 
If the input is a random vector $\mathbf{X}$ with mean value $\mu_{\mathbf{X}}$ and covariance matrix $C_{\mathbf{X}}$, and the Taylor expansion is carried out around the mean value $\mu_{\mathbf{x}}$ of $\mathbf{X}$, due to the linearity of the operator of Equation (8), the expected value and covariance of the uncertain response $Y(\mathbf{X})$ can be approximated by:

$$
\begin{gathered}
E[Y]=\mu_{Y}(\mathbf{x})+y\left(\mu_{\mathbf{x}}\right) \\
C_{Y}=\left.\nabla y\right|_{\mathbf{x}=\mu_{\mathbf{X}}} \cdot C_{\mathbf{X}} \cdot\left(\left.\nabla y\right|_{\mathbf{x}=\mu_{\mathbf{X}}}\right)^{T}
\end{gathered}
$$

This corresponds to carrying a First-Order Second-Moment (FOSM) analysis (Patelli et al., 2010). In the case of uncorrelated input random variables, the variance of the response can be expressed as:

$$
\sigma_{Y}^{2}=\sum_{i=1}^{N}\left(\left.\frac{\partial y}{\partial x_{i}}\right|_{\mathbf{x}=\mu_{\mathbf{X}}}\right)^{2} \cdot \sigma_{X_{i}}^{2}
$$

According to this expression, the response variance can be seen as the sum of the contributions of the different components of the input vector, weighted by the square of the local derivative. By dividing both members of Equation (11) by $\sigma_{Y}^{2}$, one obtains:

$$
1=\sum_{i=1}^{n_{x}}\left(\left.\frac{\partial y}{\partial x_{i}}\right|_{\mathbf{x}=\mu \mathbf{X}}\right)^{2} \cdot \frac{\sigma_{X_{i}}^{2}}{\sigma_{Y}^{2}}=\sum_{i=1}^{n_{x}} \eta_{X_{i}}^{2}
$$

where $\eta_{X_{i}}^{2}$ denotes the relative importance of the $i$-th component of $\mathbf{X}$ to the output variance. It is noteworthy the sum is equal to one only in the case of a linear model and uncorrelated input random variables, or more in general for additive models. Moreover, the sensitivity indices defined in Eq. (12) are hybrid local-global, because $\frac{\partial y}{\partial x_{i}}$ is evaluated locally, while $\sigma_{Y}$ and $\sigma_{X_{i}}$ are global. 
If the interest is in the effect of more substantial variations of $\mathbf{x}$ in its entire range of existence, then global sensitivity analysis (GSA) must be employed. GSA is based on the analysis of variance (ANOVA), i.e., on the evaluation of the relative contribution of the input to the output uncertainty, measured by the variance $V$ analysis (Patelli et al., 2010). In this paper, reference is made to the method of Sobol for GSA (Sobol', 1993). In order to illustrate the method, the inputs are assumed independently and uniformly distributed within the $N$-dimensional unit hypercube, i.e. $\mathbf{x} \in \Omega_{n_{x}}=[0,1]^{n_{x}}$. This incurs no loss of generality because any input space can be transformed onto this unit hypercube. Sobol (Sobol', 1993) has shown that $f(\mathbf{x})$ can be decomposed into the sum of terms of increasing dimension:

$$
f(\mathbf{x})=f_{0}+\sum_{i=1}^{n_{x}} f_{i}+\sum_{1 \leq i<j \leq n_{x}} f_{i j}+\ldots+f_{1,2, \ldots n_{x}}
$$

where $f_{i}=f_{i}\left(x_{i}\right), f_{i j}=f_{i j}\left(x_{i}, x_{j}\right)$, and so on.

If each term in the expansion above is square integrable and has zero mean, i.e.:

$$
\int_{0}^{1} f_{12 \ldots s}\left(x_{1}, x_{2}, \ldots, x_{s}\right) d x_{1} d x_{2} \ldots d x_{s}=0 \quad s=1,2, \ldots, n_{x}
$$

then all the terms of the decomposition are orthogonal in pairs, and the terms of the series can be univocally calculated using the conditional expectations of the model output $y$ as:

$$
\begin{aligned}
& f_{0}=\int_{\Omega_{N}} f(\mathbf{x}) d \mathbf{x} \\
& f_{i}\left(x_{i}\right)=\int_{0}^{1} \ldots \int_{0}^{1} f(\mathbf{x}) d \mathbf{x}^{i}-f_{0}=E\left(y \mid x_{i}\right)-E(y) \\
& f_{i j}\left(x_{i}, x_{j}\right)=\int_{0}^{1} \ldots \int_{0}^{1} f(\mathbf{x}) d \mathbf{x}^{i j}-f_{i}\left(x_{i}\right)-f_{j}\left(x_{j}\right)-f_{0}=E\left(y \mid x_{i}, x_{j}\right)-f_{i}\left(x_{i}\right)-f_{j}\left(x_{j}\right)-f_{0}
\end{aligned}
$$

and so on, where $d \mathbf{x}=d x_{1} d x_{2} \ldots d x_{n_{x}}$ and $d \mathbf{x}^{i}$ denotes the product $d \mathbf{x}$ without $d x^{i}$ and $d \mathbf{x}^{i j}$ denotes the product $d \mathbf{x}$ without $d x^{i}$ and $d x^{j}$. 
From Equation (15), it can be seen that $f_{i}\left(x_{i}\right)$ is the effect of varying $x_{i}$ alone, known as the main effect of $x_{i}$, and $f_{i j}\left(x_{i}, x_{j}\right)$ is the effect of varying $x_{i}$ and $x_{j}$ simultaneously, minus the effect of their individual variations, known as second-order interaction. Higher-order terms have analogous definitions.

Now, further assuming that $f(\mathbf{x})$ is square-integrable, the functional decomposition may be squared and integrated over the whole domain $\Omega_{N}$ to give the total variance of the output:

$$
V=\int_{\Omega_{N}} f^{2}(\mathbf{x}) d \mathbf{x}-f_{0}^{2}
$$

The partial variances can also be obtained as:

$$
\begin{aligned}
V_{i} & =\int_{0}^{1} f_{i}^{2}\left(x_{i}\right) d x_{i} \\
V_{i j} & =\int_{0}^{1} \int_{0}^{1} f_{i j}^{2}\left(x_{i}, x_{j}\right) d x_{i} d x_{j}
\end{aligned}
$$

and so on.

The total variance of the response can be accordingly expressed as:

$$
V=\sum_{i=1}^{N} V_{i}+\sum_{1 \leq i<j \leq n_{x}} V_{i j}+\ldots+V_{12 \ldots n_{x}}
$$

The variances of the terms in the decomposition above are the measures of importance being sought. In particular, by dividing $V_{i}$ and $V_{i j}$ by the unconditional variance $V$, we obtain respectively the first-order and second-order sensitivity indices:

$$
\begin{aligned}
& S_{i}=\frac{V_{i}}{V}=\frac{V\left[E\left(y \mid x_{i}\right)\right]}{V} \\
& S_{i j}=\frac{V_{i j}}{V}=\frac{V\left[E\left(y \mid x_{i}, x_{j}\right)\right]-V\left[E\left(y \mid x_{i}\right)\right]-V\left[E\left(y \mid x_{j}\right)\right]}{V}
\end{aligned}
$$

While $S_{i}$ estimates the expected fraction of the output variance that could be removed if the true value of $x_{i}$ exists and is known, $S_{i j}$ measures the joint effect of $x_{i}$ and $x_{j}$ on $V$. A total 
sensitivity index $S_{T i}$ can also be defined for each parameter $x_{i}$ that is the sum of all effects (first and higher orders) involving variable $x_{i}$, i.e. the sum of all the sensitivity indices having $i$ in their index. This index represents the total effect of the uncertainty of the $i$-th input parameter on the output variance.

By dividing Equation (18) by $V$, the following relation between the sensitivity indices is obtained:

$$
1=\sum_{i=1}^{N} S_{i}+\sum_{1 \leq i<j \leq n_{x}} S_{i j}+\ldots+S_{12 \ldots n_{x}}
$$

It is noteworthy that for a model with $N$ input parameters, the total number of Sobol' indices is $2 n_{x}-1$. These indices can be estimated via Monte Carlo simulation or by means of more advanced techniques reducing the number of simulations to be carried out (Patelli et al., 2010). The time required for sensitivity analysis can be reduced significantly by employing a Kriging surrogate instead of the original FE model for expressing the input-output relationship.

The sensitivity indices can be ranked in descending order, unveiling the input parameters that contribute the most to the output variance. The variables with small indices (e.g., a total sensitivity index <0.05), having a negligible effect on the output variance, can be fixed to a constant value to simplify the model without great impact on the result. The choice of the cutoff value for considering an input parameter as significant should be chosen based on computational cost and accuracy considerations.

\section{Applications}

In this section, the proposed strategy for GSA and uncertainty propagation is applied by considering different examples. The first one is based on the analytical expression for computing the masonry strength in compression based on the strengths of the mortar and of the bricks, and it is considered mainly for testing the accuracy and efficiency of kriging metamodeling. 
The other examples consist of FE models representing single arches with different rise-to-span ratio, and of a full masonry arch bridge. The simulations and analyses required for GSA and uncertainty propagation are carried out by employing Cossan (Patelli et al., 2014), whereas the DACE toolbox (Lophaven \& Nielsen, 2002) and the ooDACE toolbox (Couckuyt et al., 2014) are used for constructing the Kriging metamodels.

\subsection{Analytical function}

The application of Kriging metamodelling and GSA is illustrated here by considering the formula of Eurocode 6 (2005) for the compressive strength of masonry $f_{c k}$, which is expressed based on the values of the compressive strength of the masonry unit $f_{c u}$ and of the mortar $f_{c m}$ as:

$$
f_{c k}=K f_{c u}^{0.7} f_{c m}^{0.3}
$$

where $K$ is a constant which can be assumed equal to 0.55 for masonry with solid units and general purpose mortar (Eurocode 6, 2005).

Milani and Benasciutti (2010) assessed the accuracy of the response surface technique as a surrogate model of Equation (21) for the purpose of evaluating the probabilistic distribution of $f_{c k}$, under the assumption that $f_{c u}$ and $f_{c m}$ are independent Gaussian random variables, with mean and standard deviation estimated from some experimental data reported by Brencich and Gambarotta (2005) for eccentrically compressed masonry triplets. In particular, the mean values of $f_{c u}$ and $f_{c m}$ are respectively 19.91 MPa and 14.72 MPa, whereas the standard deviations are respectively $2.845 \mathrm{MPa}$ and $0.566 \mathrm{MPa}$.

A Kriging metamodel $\hat{f}_{K}\left(f_{c u}, f_{c m}\right)$ approximating the analytical function of Equation (21) is constructing by employing Sobol sampling (Sobol, 1976) for the design of experiments, assuming a range of variation for the input parameters within three standard deviations from the 
mean. Different increasing values of the number of observations $n_{s}$ between 5 and 100, and different types of correlation functions and orders of the polynomial are considered. For each metamodel, the optimal values of $\boldsymbol{\theta}$ and $\sigma^{2}$ are evaluated using the Dace Toolbox (Lophaven et al., 2002). The metamodel accuracy is evaluated via the leave-one-out cross-validation technique, by computing the coefficient of determination $Q^{2}$ of Equation (7). Figure 4 reports the values of $Q^{2}$ obtained for the different choices adopted for constructing the Kriging metamodel. In general, the metamodel accuracy increases by increasing $n_{s}$, with a very slow rate if more than 50 samples are employed. Moreover, using a first-order polynomial rather than a constant term (zero-order polynomial) reduce slightly $Q^{2}$ for a small size of the training set, while the use of a linear correlation model rather than a Gaussian one does not change the estimate accuracy.

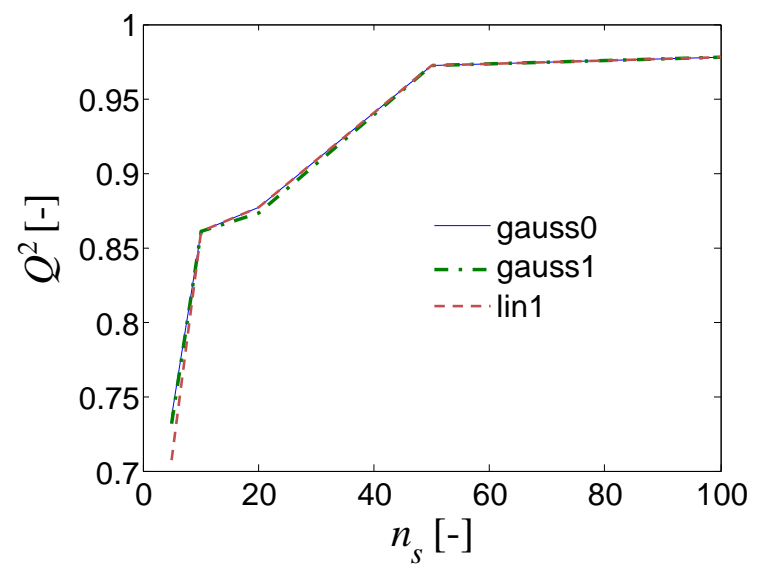

Figure 4. Coefficient of determination $Q^{2}$ vs. number of samples used to train the metamodel $n_{s}$ for different types of kriging metamodels. gauss $0=$ Gaussian correlation function and 0 degree polynomial, gauss $1=$ Gaussian correlation function and 1 st degree polynomial, lin $1=$ linear correlation function and $1^{\text {st }}$ degree polynomial.

A response surface approximation $\hat{f}_{K}\left(f_{c u}, f_{c m}\right)$ based on a quadratic polynomial is also constructed, by considering the same set of samples used for the Kriging metamodel. MCS is then carried out to propagate the uncertainty of the input parameters through the analytical function and the surrogate, and thus estimate the probability distribution of $f_{c k}$. In particular, 50000 samples of $f_{c u}$ and $f_{c m}$ are employed, given the very low computational time required by each 
analysis. The kriging metamodel considered here is based on a first order polynomial approximation and a Gaussian correlation function. A number of 20 observations is considered for both Kriging and the Response surface metamodels. Figure 5a shows the empirical cumulative distribution function (ECDF) of $f_{c k}$ according to the analytical models and the metamodels, and Figure $5 \mathrm{~b}$ a relevant zoom in the range of values of $f_{c k}$ between 6.5 and $7 \mathrm{MPa}$, corresponding to very low probability of not exceedance. It can be seen that both the metamodels provide a very good estimate of the probabilistic distribution of $f_{c k}$, despite the low number of support points employed. The Kriging metamodel is more accurate than the RS metamodel, and provides an estimate of the ECDF closer to the estimate obtained by employing the analytical model. This result is general and can be observed also by repeating the metamodel calibration and MCS for different sets of samples.

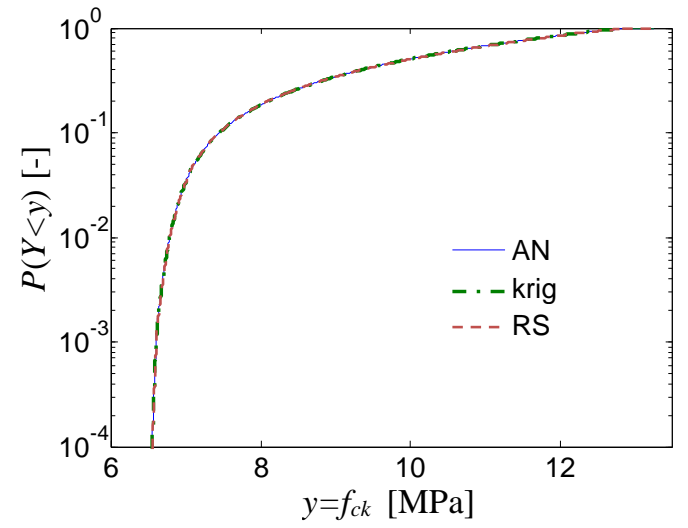

(a)

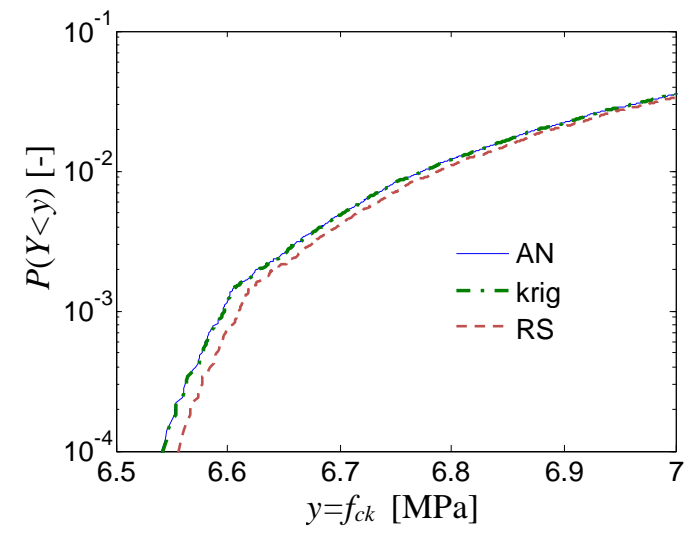

(b)

Figure 5. (a) ECDF of the output according to the analytical model, the kriging metamodel and the RS model and (b) relevant zoom. 


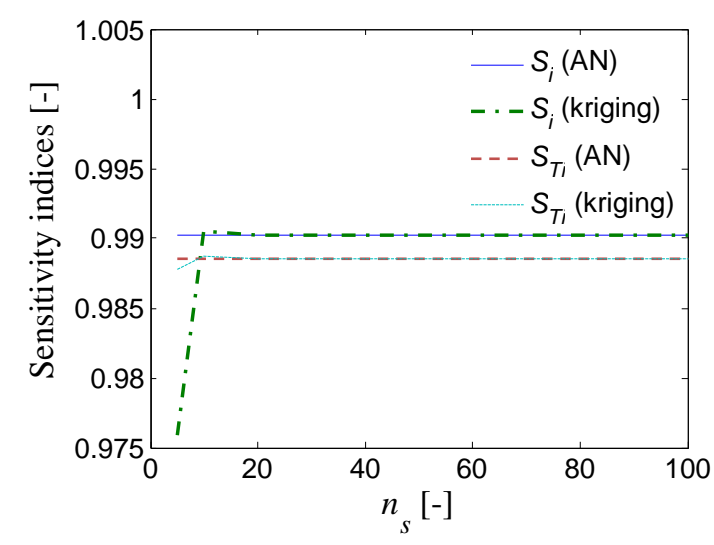

(a)

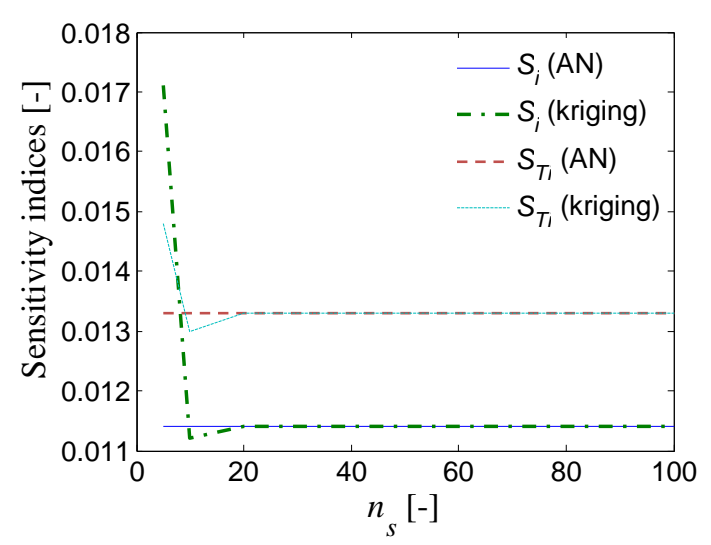

(b)

Figure 6. (a) First order and total sensitivity indices according to the analytical and kriging metamodel for (a) $f_{c u}$ and (b) $f_{c m}$.

The first order index and total sensitivity indices of $f_{c u}$ and $f_{c m}$ are evaluated by employing the method of Saltelli (Saltelli \& Chan, 2000) in conjunction with the kriging metamodel. In particular, the mean estimate $\hat{y}(\mathbf{x})$ according to Equation (4) is used to evaluate $f_{c k}$ given the samples of $f_{c u}$ and $f_{c m}$. Figure 6 plots the variation of these indices with respect to the number of observations used to construct the metamodel. In the same figure, the estimate of the sensitivity indices obtained by considering the analytical function are reported for comparison purposes.

First of all, it can be observed that if a number of support points higher than 20 is considered, the kriging metamodel yields the same estimate of the sensitivity indices as the true analytical model. Moreover, $f_{c u}$ has a very high influence on the variance of $f_{c k}$, whereas the influence of $f_{c m}$ and of the interaction term is negligible. The difference between the first order and the total sensitivity index is very low for both the input variables, and the sum of the two first order sensitivity indices is equal to 1 . The values of the sensitivity indices evaluated by means of LSA according to Equation (12) are $\eta_{f_{c u}}=0.992$, and $\eta_{f_{c m}}=0.093$. Thus, while the sensitivity index for $f_{c u}$ is similar to that obtained via GSA, the sensitivity index for $\eta_{f_{c m}}$ is much higher. 


\subsection{Masonry arch}

This numerical example is based on an experimental test carried out at the University of Salford (Melbourne et al., 2007). The analysed structure consists of a $3 \mathrm{~m}$ span multi-ring brick masonry square arch constructed using the stretcher bonding method with a 1:4 rise-to-span ratio and made up of 47 courses of bricks for the lower ring and 49 courses for the top ring. A numerical model of the arch has been previously developed by Zhang et al. (2018b) to validate the mesoscale approach for brick-masonry arches. In order to reduce the computational time of the FE analyses, the arch is described by only one set of solid elements along its width, thus disregarding the masonry texture along the width of the arch. Figure 7a shows the FE model of the arch, which is analysed by imposing increasing vertical displacements at the nodes of the loaded surface at a quarter span, whereas Figure $7 \mathrm{~b}$ illustrates the mortar interfaces between the bricks.

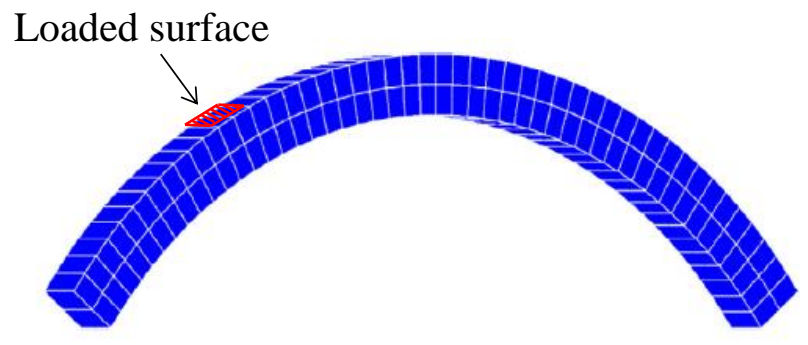

(a)

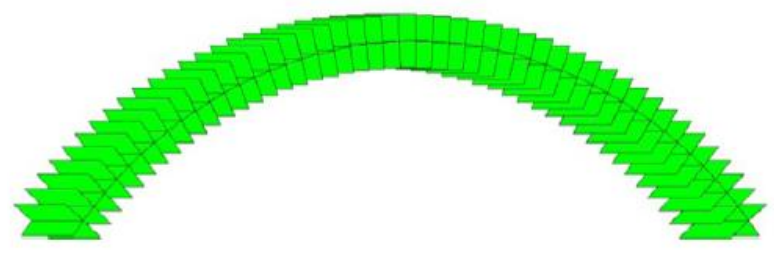

(b)

Figure 7. (a) Mesoscale model for the arch, (b) mortar interfaces.

Although dimensions and shapes of the bridge components have an important effect on the capacity (Oliveira et al. 2010, Cavalagli et al. 2017), in this study they are assumed as deterministic, as the focus of this research is on the identification of the critical mesoscale material parameters which should be determined via suitable material testing to achieve accurate response predictions. Moreover, it is envisaged that the uncertainty in the definition of the geometrical characteristics of the analysed structure can be minimised through an accurate survey 
(de Arteaga and Morer 2021, Riveiro et al. 2013). The kriging metamodel is built by considering different input parameters related to the mechanical behaviour of the bricks and of the interfaces that could potentially affect the response of the arch under vertical loads. Table 1 lists these parameters together with their range of variation. It is noteworthy that the parameters are assumed to be independent. While this can be a limitation, leading to an underestimation of the interaction effects, accounting for the correlation of the parameters would require an extension of the GSA methodology followed here (Li et al., 2010) which is out of the scope of the present study and the subject of future investigations. Nevertheless, in order to introduce some correlation between the tangent and normal stiffness in the mortar interfaces, the ratio $k_{H} / k_{N}$ rather than $k_{H}$ is considered as input parameter. Moreover, for describing the tensile yield behaviour, the ratio $s_{0}$ between the actual tensile resistance of the interfaces $\sigma_{t 0}$ and the maximum value according to the Mohr-Coulomb criterion (i.e., $\left.c_{0} / \tan \phi_{0}\right)$ is specified. This prevents the consideration of physically inconsistent values of the tensile resistance. A very wide range of variation is considered for the brick Young modulus $E_{\mathrm{b}}$, to describe the case of bricks with very poor mechanical properties and high strength bricks such as engineering bricks. The range of variation of $G_{f, I}$ and $G_{f, I I}$ is taken from Lourenço (1996). The parameters describing the compressive behaviour of masonry are assumed fixed and not explicitly considered for building the metamodel since compressive failure of masonry is not envisaged. In particular, a value of $24.5 \mathrm{MPa}$ is assumed for the interface compressive limit. The brick density is also assumed constant and equal to $1900 \mathrm{~kg} / \mathrm{m}^{3}$.

Table 1. Input parameters of the arch metamodel, relevant range of variation, mean and cov of the lognormal distribution.

\begin{tabular}{|c|c|c|c|c|}
\hline \multirow{3}{*}{ Element } & Type & Range & Mean & CoV \\
\hline \multirow{3}{*}{ Brick } & Elastic modulus $E_{\mathrm{b}}\left[\mathrm{N} / \mathrm{mm}^{2}\right]$ & $5000-40000$ & 16000 & 0.27 \\
\cline { 2 - 5 } & Poisson's ratio $\mathrm{v}_{b}[-]$ & $0.1-0.4$ & 0.15 & 0.2 \\
\hline \multirow{3}{*}{ Mortar-Brick interface } & Normal stiffness $k_{N}\left[\mathrm{~N} / \mathrm{mm}^{3}\right]$ & $3-1000$ & 90 & 0.27 \\
\cline { 2 - 5 } & Tangent to normal stiffness ratio $k_{H} / k_{N}\left[\mathrm{~N} / \mathrm{mm}^{3}\right]$ & $0.3-0.5$ & 0.444 & 0.2 \\
\cline { 2 - 5 } & Cohesion $c_{0}\left[\mathrm{~N} / \mathrm{mm}^{2}\right]$ & $0.0001-1.8$ & 0.4 & 0.38 \\
\hline
\end{tabular}




\begin{tabular}{|c|c|c|c|c|}
$\mid$ Friction angle $\tan \phi_{0}[-]$ & $0.0001-1.5$ & 0.5 & 0.2 \\
\cline { 2 - 5 } & Tensile resistance parameter $s_{0}\left[\mathrm{~N} / \mathrm{mm}^{2}\right]$ & $0-1$ & 0.325 & 0.2 \\
\cline { 2 - 5 } & Fracture energy for tension mode $G_{f I}\left[\mathrm{~N} / \mathrm{mm}^{2}\right]$ & $0.005-0.02$ & 0.012 & 0.2 \\
\cline { 2 - 5 } & Fracture energy for tension mode $G_{f I I}\left[\mathrm{~N} / \mathrm{mm}^{2}\right]$ & $0.01-0.25$ & 0.125 & 0.2 \\
\hline
\end{tabular}

The kriging metamodel is constructed by considering as output variables the peak load capacity per unit arch width $p_{c}$ and the secant stiffness to $40 \%$ of the peak load capacity $k_{s e c}$. The input samples are generated using the Sobol sequence by considering a maximum number of $n_{s}$ $=500$ observations. For each input parameter combination, a FE analysis is performed in ADAPTIC (Izzuddin, 1991) to obtain the full load-displacement curve relating the reaction forces at the nodes subjected to the imposed displacement history and the vertical displacement of a node at the intrados of the arch barrel located at quarter span. Then, these curves are postprocessed to identify the values of $p_{c}$ and of $k_{s e c}$. Figure 8a shows the most recurrent failure mode of the arch, corresponding to the formation of a mechanism consisting of four hinges, whereas Figure $8 \mathrm{~b}$ shows the corresponding contour plot of the damage in tension parameter.

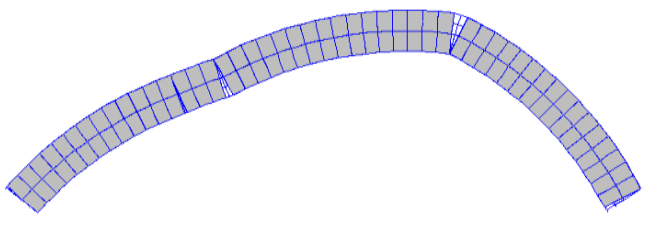

(a)

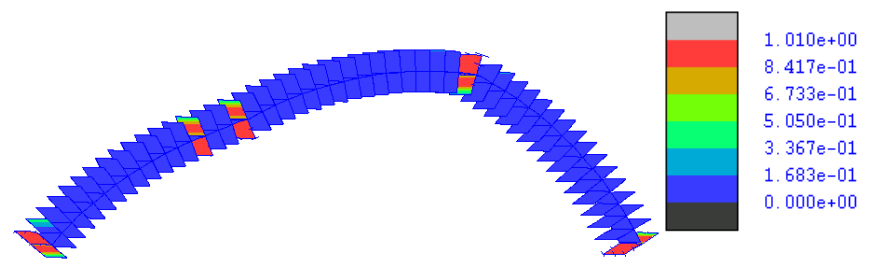

(b)

Figure 8. (a) Most recurrent failure mode and (b) corresponding contour plot of the damage parameter in tension.

The set of support points generated via Sobol sampling (Sobol, 1976) yield very different collapse behaviours of the arch, the most interesting of which are reported in Figure 9. For example, the mechanism of Figure 9a and Figure 9c correspond to low values of the friction angle of the mortar joints $\left(\tan \phi_{0}=0.02\right.$ and $\tan \phi_{0}=0.08$ respectively). The mechanism of Figure $9 \mathrm{~b}$ corresponds to high values of the tensile resistance, and low values of the cohesion and of the friction angle compared to the reference case $\left(s_{0}=0.86, \tan \phi_{0}=0.16, c_{0}=0.20 \mathrm{MPa}\right)$. Finally, 
the mechanism of Figure 9d corresponds to very high values of the cohesion of the mortar interfaces $\left(s_{0}=0.56, \tan \phi_{0}=0.61, c_{0}=1.52 \mathrm{MPa}\right)$.

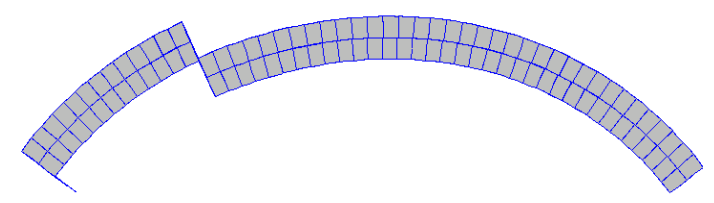

(a)

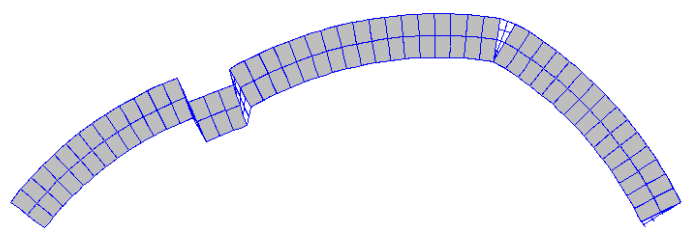

(c)

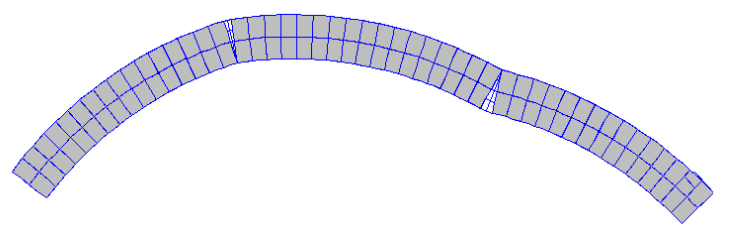

(b)

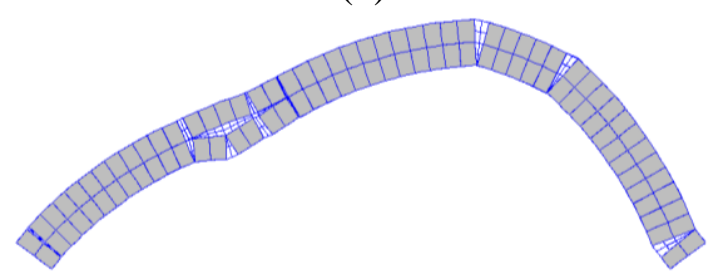

(d)

Figure 9. Different failure modes observed for the combination of the input parameters.

The values of $p_{c}$ vary in the range between $0.0023 \mathrm{kN} / \mathrm{mm}$ and $0.56 \mathrm{kN} / \mathrm{mm}$, whereas the values of $k_{s e c}$ in the range between $0.0071 \mathrm{kN} / \mathrm{mm}^{2}$ and $0.6176 \mathrm{kN} / \mathrm{mm}^{2}$. A kriging metamodel is fitted to the input-output data by employing first-order polynomial for the global trend and the Gaussian correlation model of Equation (3) for $R\left(\mathbf{x}, \mathbf{x}^{\prime}\right)$. The Dace Toolbox (Lophaven et al., 2002) is used to find the optimal values of $\boldsymbol{\theta}$ and $\sigma^{2}$.

Figure 10 illustrates the variation of the coefficient of determination $Q^{2}$ of the kriging metamodels for $p_{c}$ and $k_{s e c}$ with the number of training points. It can be seen that the values of $Q^{2}$ increase for increasing $n_{s}$, and that for a given value of $n_{s}$, the $Q^{2}$ corresponding to $k_{s e c}$ is higher than that of $p_{c}$. Moreover, with 500 training points a very high level of accuracy is achieved. 


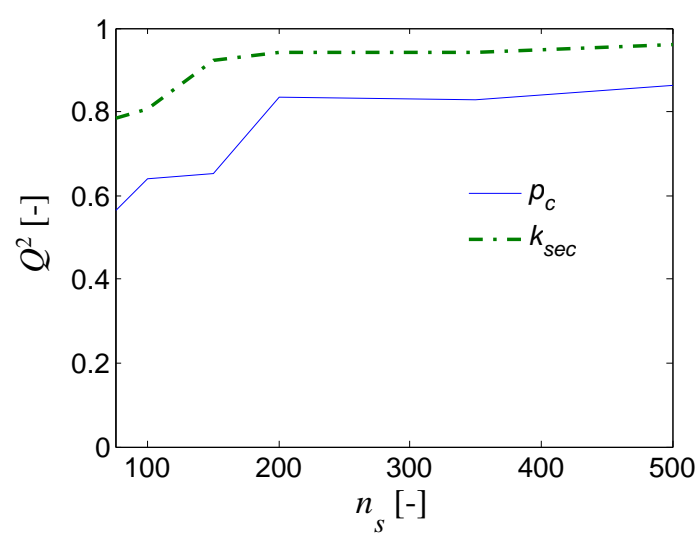

Figure 10. Variation with the number of training points of $\mathrm{Q}^{2}$ of the metamodel for the collapse load $p_{c}$ and the secant stiffness $k_{\text {sec }}$.

Figure 11 and Figure 12 show the first order sensitivity indices of the input parameters obtained considering respectively the collapse load and the secant stiffness as output parameters. These indices have been evaluated employing the method of Saltelli (Saltelli and Chan, 200) in conjunction with kriging metamodel fitted by considering 250 and 500 support points. In general, it can be observed that the estimates of the indices is not significantly affected by the number of support points considered, i.e., 250 samples are enough to achieve reliable estimates of the indices.

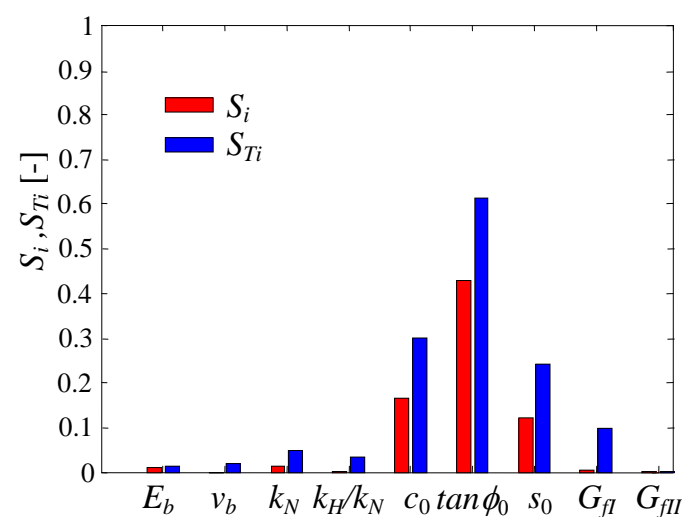

(a)

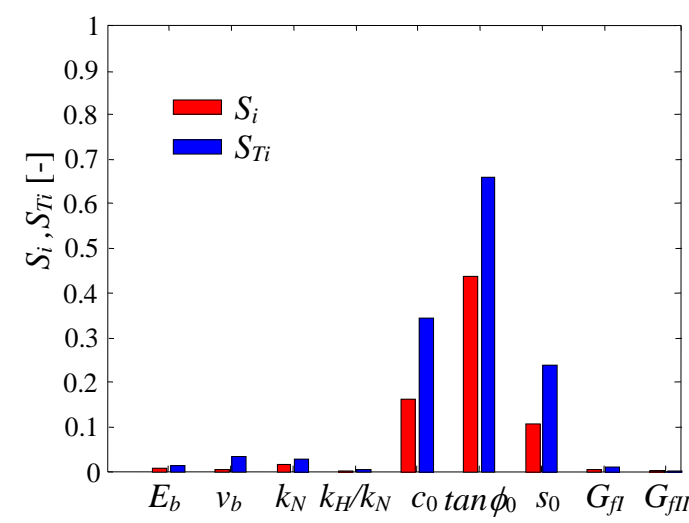

(b)

Figure 11. First order and total sensitivity indices for the collapse load obtained by considering (a) 250 samples and (b) 500 samples for training the metamodel. 


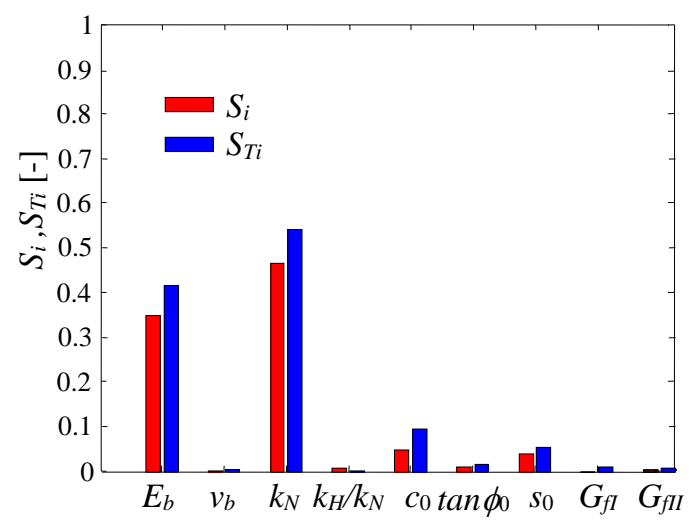

(a)

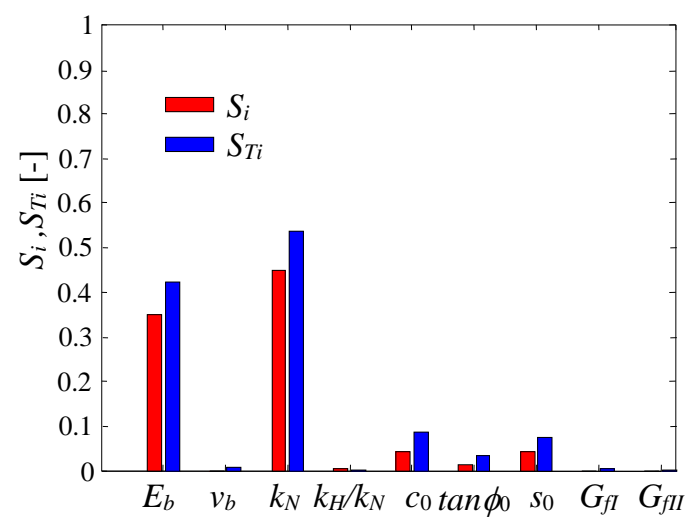

(b)

Figure 12. First order and total sensitivity indices of the secant stiffness obtained by considering (a) 250 samples and (b) 500 samples for training the metamodel.

As expected, the parameters that influence the most the collapse load (Figure 11) are those related to the resistance of the mortar interfaces. Similar results have been observed in Zhang et al. (2016) based on an extensive parametric study encompassing large variations of many parameters related to the mortar interface behaviour. The most influential parameter is the friction angle $\tan \phi_{0}$, followed by the cohesion $c_{0}$ and by the parameter $s_{0}$, related to the tensile resistance. It is noteworthy that the tensile resistance of the mortar interfaces is also influenced by $c_{0}$ and $\tan \phi_{0}$, and this together with the fact that in most of the cases failure of the arch occurs due to formation of flexural hinges explains why these parameters are characterised by very high sensitivity indices. With reference to the secant stiffness (Figure 12), the most influential parameters in order of importance are the normal stiffness of the mortar interfaces $k_{N}$, the brick Young modulus $E_{b}$, followed by the parameters describing the interface resistance, i.e., $c, s$, $\tan \phi 0$. While the influence of the first two parameters is obvious, the fact that the interface resistance parameters influence the stiffness of the bridge is because a secant stiffness is considered, which depends on the bridge capacity.

For the purpose of performing uncertain propagation, the input parameters employed for constructing the metamodel are assumed as independent random variables with a lognormal 
distribution. The mean and coefficient of variation of the distributions are reported in Table 1. The values adopted for many of the parameters are arbitrary, due to lack of available statistical data, but they are similar to those employed in other studies (Moreira et al., 2016; Moreira et al., 2017). In particular, the coefficient of variation (CoV) of $E_{b}$ and $k_{N}$ are based on the works of (De Felice and De Santis, 2010; Kaushik et al. 2007), that of tan $\phi_{0}$ from Moreira et al. (2017), and that of $c_{0}$ is taken from Jie et al. (2017).

A Monte Carlo simulation is carried out in Cossan (Patelli et al. 2014) by generating 20000 samples of the random variables, and using the fitted metamodels to estimate the probabilistic distribution of the collapse load and of the stiffness. Figure 13a shows and compares the ECDF of the collapse load obtained by considering all the random variables as uncertain with the one obtained by considering only $c_{0}, \tan \phi_{0}, s_{0}$ as uncertain, and by fixing the other parameters to their mean values. The corresponding values of the mean and cov are reported in Table 2. It can be observed that the dispersion of the collapse load is quite high, and slightly higher than that of most of the input random variables. Moreover, considering only the parameters that influence the most the collapse load allows an accurate estimation of the mean and dispersion. Table 2 reports also the results FOSM, which provides good predictions of the mean collapse load, and quite good estimates of dispersion.

With reference to the secant stiffness, the ECDFs obtained accounting for all the random variables and assuming only $E_{b}, k_{N}, c_{0}, \tan \phi_{0}, s_{0}$ as uncertain are plotted in Figure $13 \mathrm{~b}$. Table 2 provides the corresponding values of the mean and coefficient of variation. Considering the reduced set of uncertain variable yields again very accurate estimates of the mean value, but it underestimates significantly the dispersion. According to the results shown in Table 2, FOSM provides quite good estimates of both the mean value and the dispersion.

a)

b) 

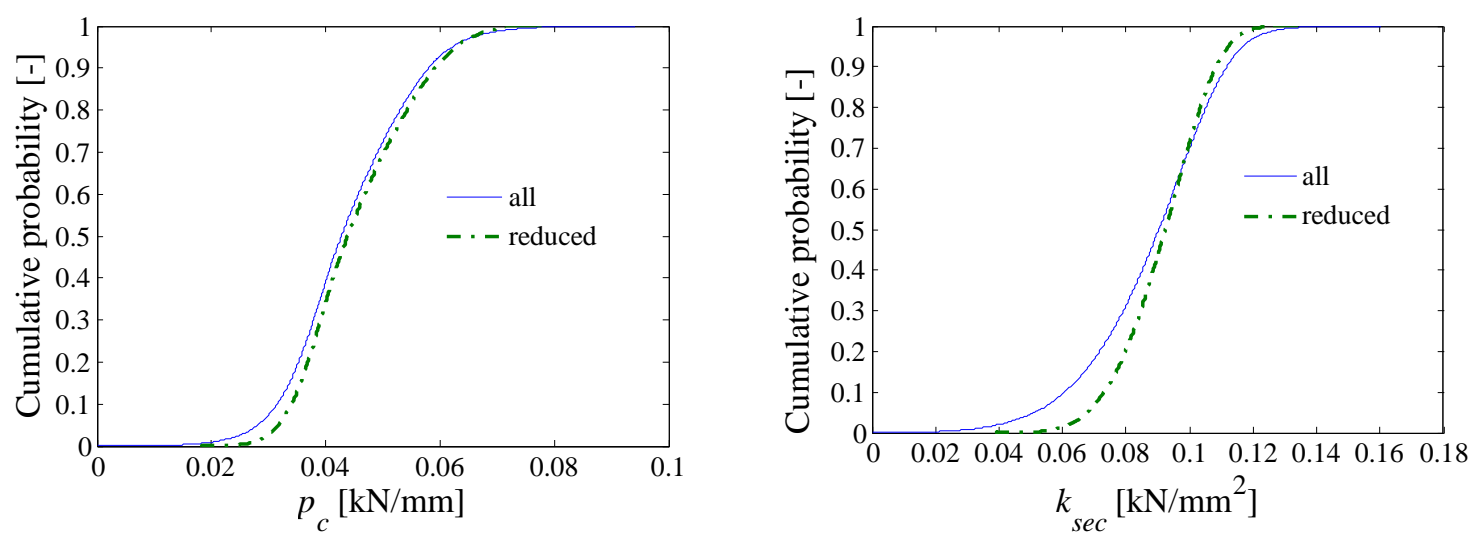

Figure 13. ECDF of the collapse load (a) and of the secant stiffness (b) obtained by considering all the parameters of Table 2 as uncertain and only few parameters as uncertain.

Table 2. Mean and cov of the collapse load and of the secant stiffness obtained via MCS and via FOSM.

\begin{tabular}{|c|c|c|c|c|}
\cline { 2 - 5 } \multicolumn{1}{c|}{} & \multicolumn{2}{c|}{$p_{c}$} & \multicolumn{2}{c|}{$k_{\text {sec }}$} \\
\hline & Mean [kN/mm] & CoV & Mean [kN/mm $\left.{ }^{2}\right]$ & CoV \\
\hline MCS-Whole set & 0.044 & 0.241 & 0.0880 & 0.230 \\
\hline MCS-Reduced set & 0.045 & 0.213 & 0.0915 & 0.148 \\
\hline FOSM & 0.043 & 0.203 & 0.0899 & 0.267 \\
\hline
\end{tabular}

The study of Zhang et al. (2018b) has shown that significant changes in the response of masonry arches can be observed for different rise-to-span ratio values. For this reason, a second numerical model of a deep masonry arch is considered herein, corresponding to a rise-to-span ratio of 0.45 rather than 0.25 . GSA is repeated on the kriging metamodel of the deep arch, constructed by considering $n_{s}=500$ support points. All the other parameters of the model are the same as those of the arch analysed previously. Figure 14a shows the most recurrent failure mode of the deep arch, which is very similar to that of the shallow arch (Figure 8), and Figure $14 \mathrm{~b}$ shows the corresponding contour plot of the damage parameter in tension.

a)

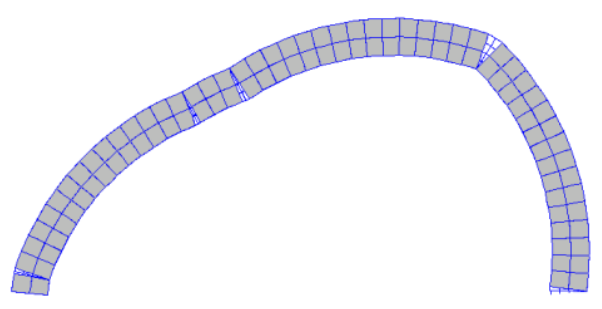

b)

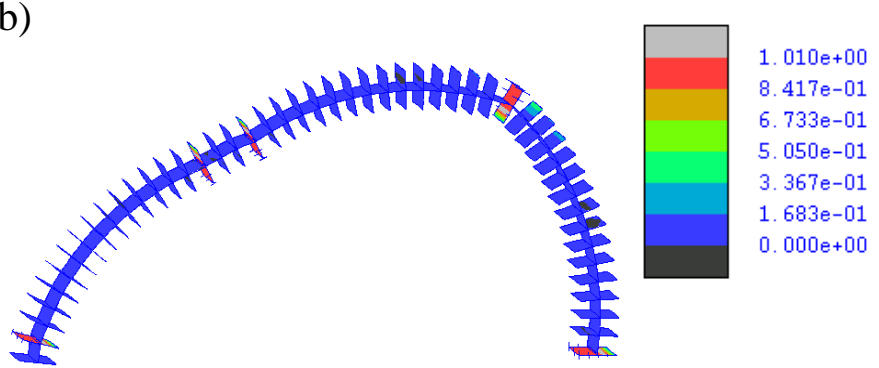

Figure 14. a) Most recurrent failure mode and b) corresponding contour plot of the damage parameter in tension. 
The results of GSA, applied by considering the collapse load and the secant stiffness as output parameters, are reported respectively Figure 15a and Figure 15b. The sensitivity indices are very similar to those of the shallow arch, reported in Figure $11 \mathrm{~b}$ and Figure $12 \mathrm{~b}$.

a)

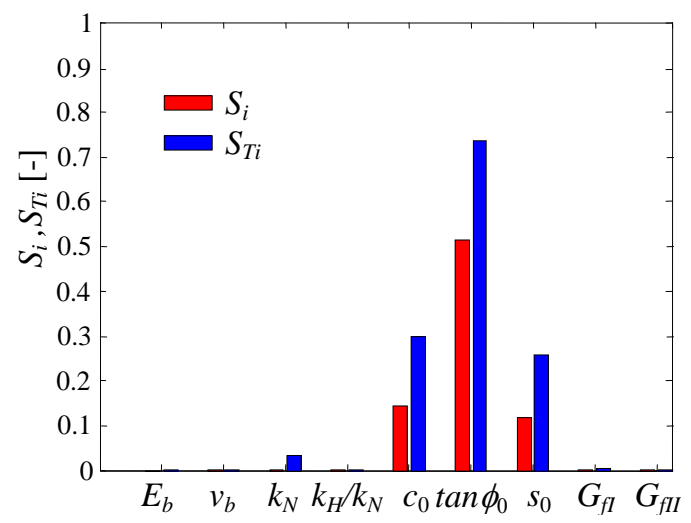

b)

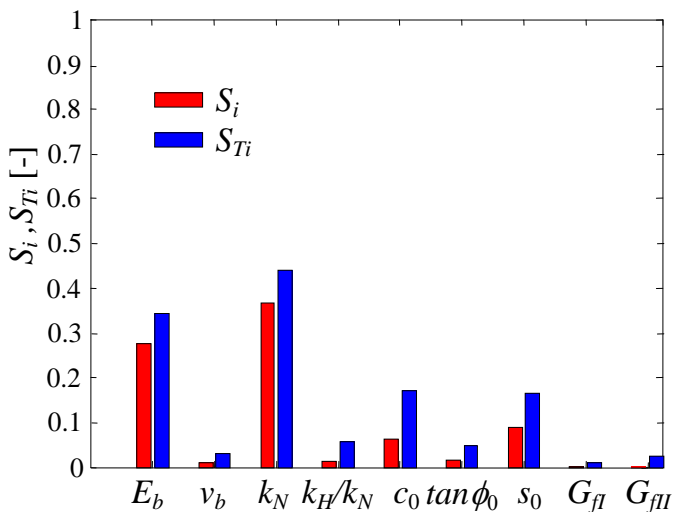

Figure 15. First order sensitivity indices for the collapse load (a) and the secant stiffness (b) of the arch with riseto-span ratio of 0.45 obtained by considering 500 samples for training the metamodel.

\subsection{Masonry arch bridge}

This numerical example is based on a single-span bridge model tested at the University of Salford (Melbourne et al., 2007). The $3 \mathrm{~m}$ span two-ring arch barrel was built according with the stretcher method in a segmental circular shape on massive concrete foundations. The arch is $215 \mathrm{~mm}$ thick and is characterised by a rise-to-span ratio of 0.25 with a springing angle of $37^{\circ}$. The spandrel and the wing walls are made of English bond brick-masonry. Full size class A engineering bricks and a 1:2:9 (cement:lime:sand) mortar were used for the brickwork, while $50 \mathrm{~mm}$ graded crushed limestone was adopted for the backfill, filling the space above the arch and between the two lateral walls (spandrel and wing walls). The bridge was tested by applying a load at quarter span until collapse. The spandrel walls are detached from the arch barrel and only provide transverse confinement to the backfill. This allows an efficient description of the test with a strip model characterised by only one set of solid elements along the width of the bridge specimen for the arch and the backfill meshes. Further information regarding the model and the validation of the experimental results can be found in Zhang et al. (2018a). 
Figure 16a shows the FE model of the bridge, including the arch barrel and the backfill, whereas Figure 16b illustrates the mortar interfaces between the bricks and the interfaces between the arch barrel extrados and the backfill. The bridge is analysed by imposing increasing vertical displacements in correspondence of the nodes belonging to the surface at the bridge extrados highlighted in Figure 16a.

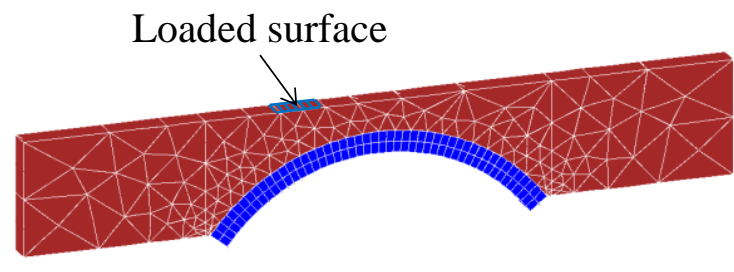

(a)

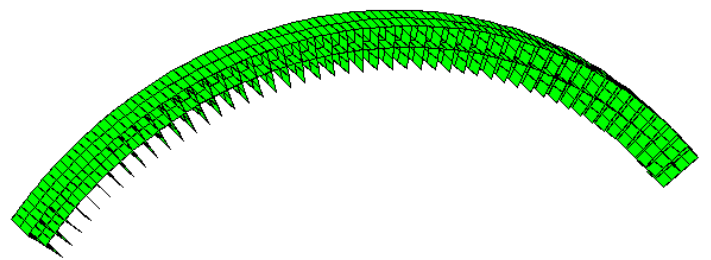

(b)

Figure 16. (a) FE strip model for masonry bridge, (b) mortar and arch barrel-backfill interfaces.

The input parameters used to build the kriging metamodel replacing the bridge FE model are reported in Table 3, and concern the mechanical properties of the bricks, the mortar interfaces, the backfill, and the arch barrel-backfill interface. Only the input properties of the bricks and mortar interfaces that have been found to be most influential from the sensitivity study of the masonry arch are considered to vary, whereas the others are kept fixed. In addition, some parameters that could have some influence on the bridge capacity such as the backfill weight have been kept fixed to limit the dimension of the metamodel. Table 3 also shows the range of variation considered for building the kriging metamodel and for the sensitivity study.

Table 3. Input parameters of the bridge metamodel, relevant range of variation, mean and cov of the lognormal distribution.

\begin{tabular}{|c|c|c|c|c|}
\hline Element & Type & Range & Mean & CoV \\
\hline \multirow{3}{*}{ Brick } & Elastic modulus $E_{b}\left[\mathrm{~N} / \mathrm{mm}^{2}\right]$ & $5000-40000$ & 35000 & 0.27 \\
\hline \multirow{4}{*}{ Mortar-Brick interface } & Normal stiffness $k_{N}\left[\mathrm{~N} / \mathrm{mm}^{3}\right]$ & $3-1000$ & 400 & 0.2 \\
\cline { 2 - 5 } & Cohesion $c_{0}\left[\mathrm{~N} / \mathrm{mm}^{2}\right]$ & $0.0001-1.8$ & 0.29 & 0.38 \\
\cline { 2 - 5 } & Friction angle $\tan \phi_{0}[-]$ & $0.0001-1.5$ & 0.5 & 0.2 \\
\cline { 2 - 5 } & Tensile resistance parameter $s_{0}[-]$ & $0-1$ & 0.345 & 0.2 \\
\hline \multirow{3}{*}{ Backfill } & Elastic modulus $E_{f}\left[\mathrm{~N} / \mathrm{mm}^{2}\right]$ & $20-500$ & 200 & 0.2 \\
\cline { 2 - 5 } & Poisson's ratio $\mathrm{v}_{f}[-]$ & $0.1-0.499$ & 0.2 & 0.2 \\
\cline { 2 - 5 } & Cohesion $c_{f}\left[\mathrm{~N} / \mathrm{mm}^{2}\right]$ & $0.0001-0.2$ & 0.001 & 0.2 \\
\hline
\end{tabular}




\begin{tabular}{|c|c|c|c|c|}
\hline & Friction angle $\tan \phi_{f}[-]$ & $0.466-1.428$ & 0.960 & 0.2 \\
\hline \multirow{4}{*}{ Arch barrel-Backfill interface } & Cohesion $c_{i}\left[\mathrm{~N} / \mathrm{mm}^{2}\right]$ & $0.0001-0.1$ & 0.001 & 0.2 \\
\cline { 2 - 5 } & Friction angle $\tan \phi_{i}[-]$ & $0.466-1.732$ & 0.6 & 0.2 \\
\cline { 2 - 5 } & Tensile resistance parameter $s_{i}\left[\mathrm{~N} / \mathrm{mm}^{2}\right]$ & $0-1$ & 0.6 & 0.2 \\
\hline
\end{tabular}

The model output variables are the peak load capacity per unit bridge width $p_{c}$ and the stiffness secant to $40 \%$ of the peak load capacity $k_{s e c}$. The same procedure employed for the masonry arch is followed here to construct the kriging metamodel, for a total of $n_{s}=1000$ support points. In particular, the kriging metamodel for $p_{c}$ has been built by considering a $0^{\text {th }}$-order polynomium for describing the global trend and a Matérn correlation function, available in the ooDACE toolbox (Couckuyt et al., 2014), for $R\left(\mathbf{x}^{l}, \mathbf{x}^{m}\right)$. The metamodel for $k_{\text {sec }}$ has been built by considering a $1^{\text {st }}$-order polynomium and a Gaussian correlation function. The values of $p_{c}$ for the various support points vary in the range between $0.0055 \mathrm{kN} / \mathrm{mm}$ and $1.36 \mathrm{kN} / \mathrm{mm}$, whereas the values of $k_{\text {sec }}$ in the range between $0.0066 \mathrm{kN} / \mathrm{mm}^{2}$ and $0.841 \mathrm{kN} / \mathrm{mm}^{2}$. The values of $Q^{2}$ corresponding to the metamodels for $p_{c}$ and $k_{s e c}$ are respectively equal to 0.80 and 0.91 .

The most recurrent failure mode of the bridge, highlighted in Figure 17a, corresponds to the formation of a mechanism consisting of four hinges whose location is very similar to that of the bare arch (Figure 8a). Figure 17b and Figure 17c show the contour plot of respectively the damage parameter in tension and the Von Mises plastic stresses in the backfill at collapse. It is observed that the backfill contributes significantly to the resistance of the bridge, spreading the loads applied on the surface of the backfill, and providing transverse resistance and passive pressure to the deformed arch. A more in-depth analysis of the mechanism involved in the bridge collapse can be found in Zhang et al. (2018a).

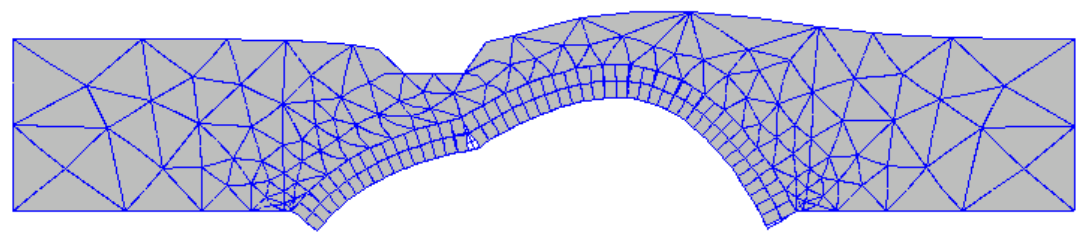




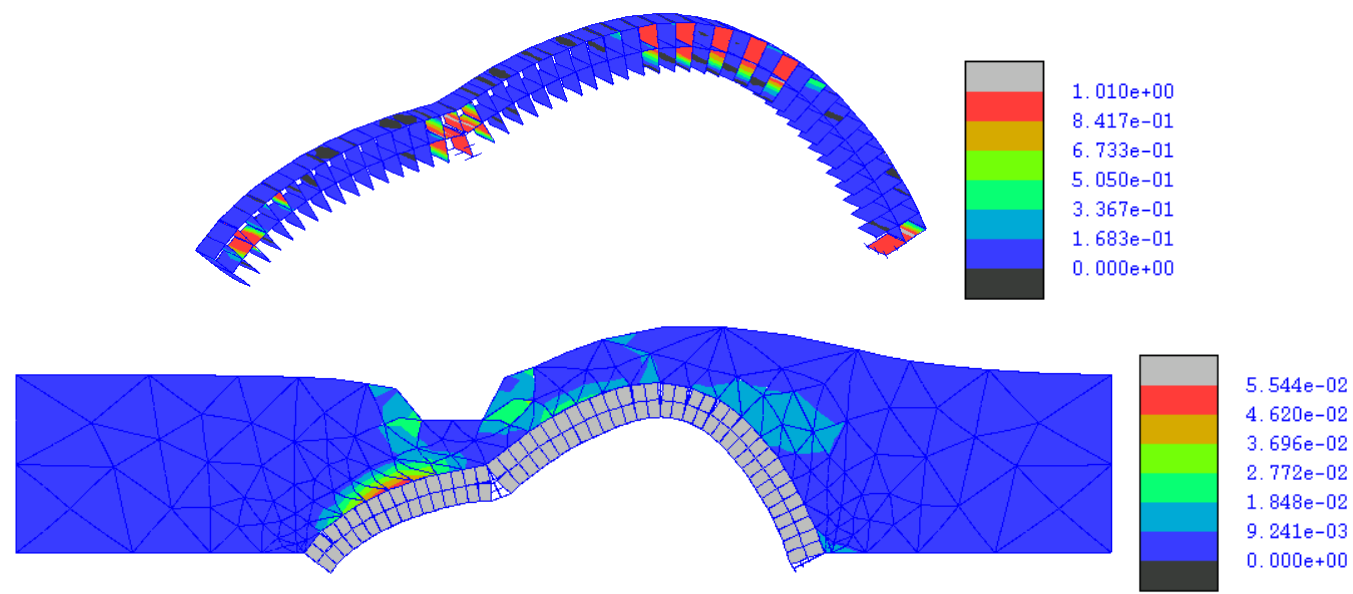

Figure 17. (a) Most recurrent failure mode, (b) corresponding contour plot of the damage parameter in tension and (c) equivalent von Mises plastic deformations in the backfill.

The application of GSA allows the derivation of the first order sensitivity indices reported in Figure 18 and Figure 19, and referring to the collapse load (Figure 18) and to the secant stiffness (Figure 19). With reference to the first, the most influential input parameters are in order of importance: the backfill cohesion $c_{f}$, the friction angle of the mortar interfaces $\tan \phi$, the young modulus of the backfill $E_{f}$, the friction angle of the backfill $\tan \phi_{f}$, and the cohesion of the mortar interfaces $c_{0}$. These results confirm the important contribution to the bridge resistance played by the backfill, and especially by the cohesion, that controls the collapse load. The number of support points employed for fitting the metamodel has a negligible influence on the sensitivity analysis results, if more than 500 samples are considered for training the metamodel. In fact, the sensitivity analysis results obtained with $n_{s}=500$ and $n_{s}=1000$ are very similar to each other.

With reference to the secant stiffness, the input parameters with highest indices of sensitivity are $E_{b}, k_{N}$, followed by $E_{f}$, and $c_{f}$ and $c_{0}$. Thus, the stiffness of the arch influences more significantly the global stiffness than the backfill stiffness. This result might be related to the fact that a very wide range of variation has been chosen for the brick Young modulus and for the mortar interfaces stiffness. The observed sensitivity analysis results are consistent with those observed 
in Zhang et al. (2018a), where only variations of the backfill properties were considered, showing that $c_{f}$ is the parameter that affects the most the bridge capacity, followed by $E_{f}$, and $\tan \phi_{f}$,.

a)

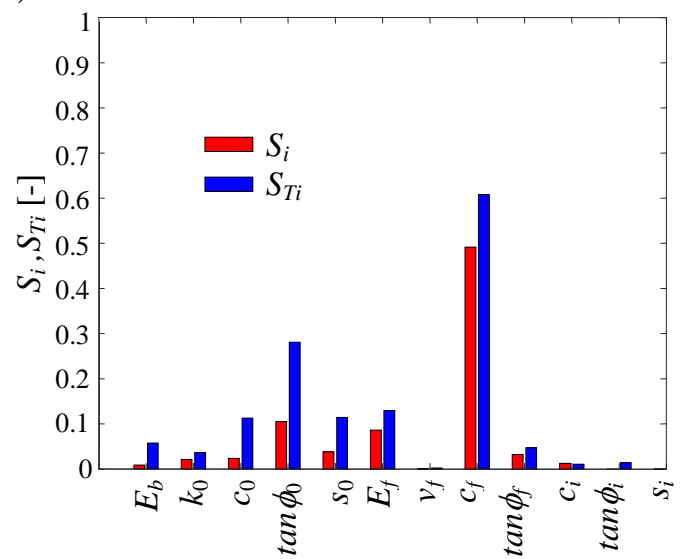

b)

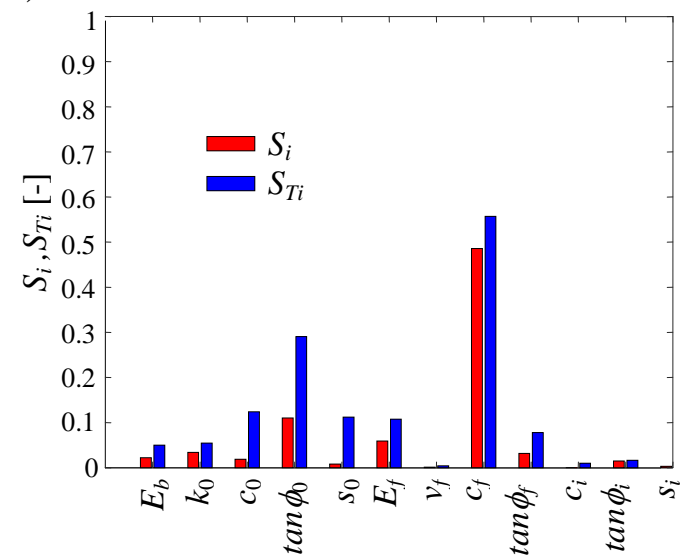

Figure 18. First order and total sensitivity index of the collapse load obtained by considering (a) 500 samples and (b) 1000 samples for training the metamodel.

a)

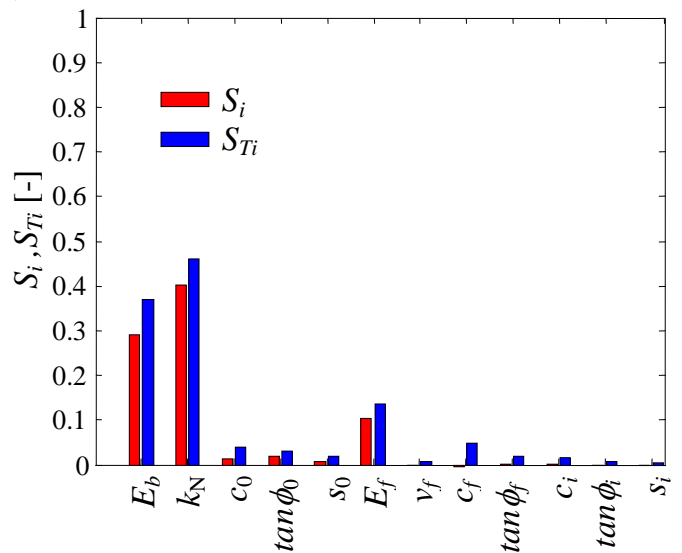

b)

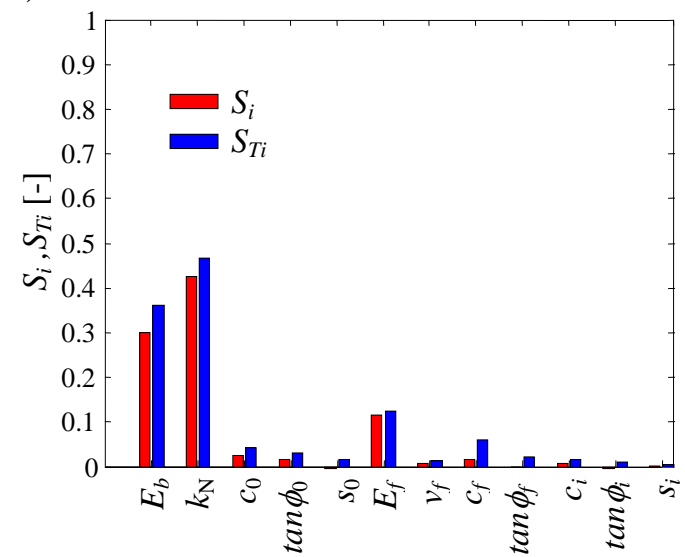

Figure 19. First order and total sensitivity index of the secant stiffness obtained by considering (a) 500 samples and (b) 1000 samples for training the metamodel.

Uncertainty propagation is carried out for this model based on the probabilistic distributions reported in Table 3. The lognormal distribution characterising the friction angle $\tan \phi_{f}$ is truncated to the value of 1.047 to avoid excessive angles of friction above $60^{\circ}$. The ECDFs of the collapse load obtained by considering all the random variables as uncertain and the one obtained by considering only $c_{f}$, $\tan \phi_{0}, E_{f}$, and $\tan \phi_{f}$ and $c_{0}$ as uncertain are illustrated in Figure 20a. The corresponding values of the mean and coefficient of variation are given in Table 4, which also 
reports the values estimated via FOSM. In general, the CoV of the collapse load is quite high, and close to 0.4. Neglecting the uncertainty of the least influential parameters does not affect the estimate of the mean and of the dispersion. On the other hand, FOSM provides good estimates of the first moment of the collapse load, but overestimates the second one.

The ECDFs of the secant stiffness, plotted in Figure 20b, are estimated by accounting for all the random variables and by assuming only $E_{b}, k_{N}, E_{f}$, and $c_{f}$ and $c_{0}$ as uncertain. The $\mathrm{CoV}$ is lower than that of the collapse load. Considering the reduced set of uncertain variable yields again quite good estimates of the mean value, but underestimates the dispersion. According to the results shown in Table 4, FOSM slightly overestimates the mean value of the secant stiffness, while it underestimate the dispersion.

a)

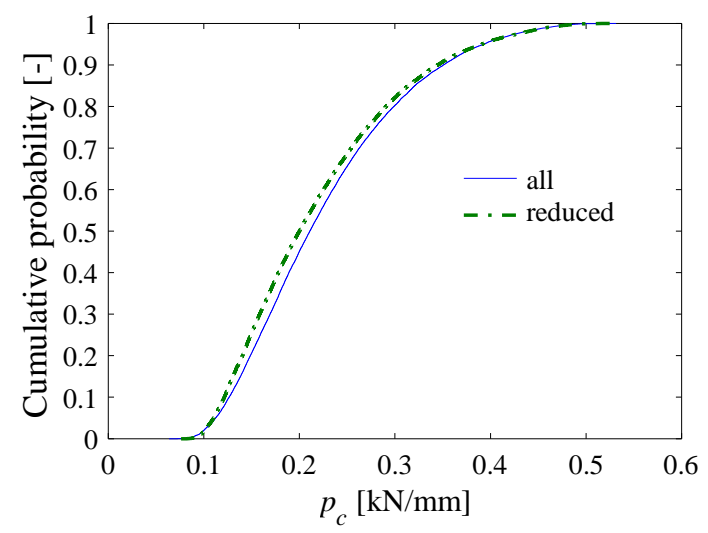

b)

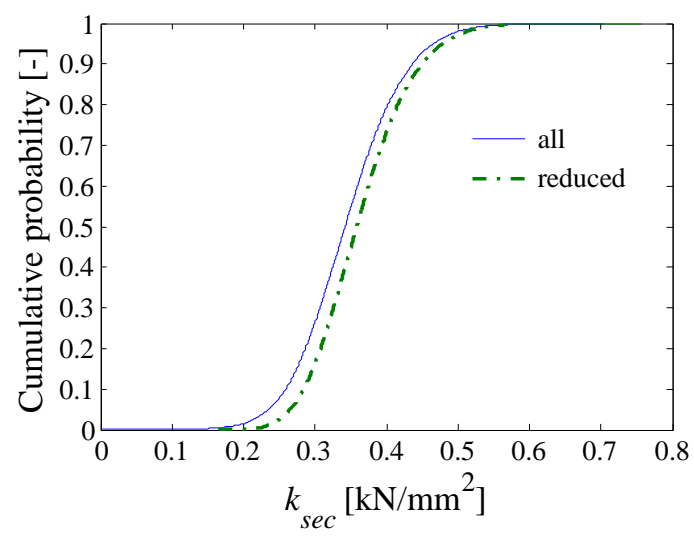

Figure 20. ECDF of the collapse load (a) and of the secant stiffness (b) obtained by considering all the parameters of Table 3 as uncertain and only few parameters as uncertain.

Table 4. Mean and cov of the collapse load and of the secant stiffness obtained via MCS and via FOSM.

\begin{tabular}{|c|c|c|c|c|}
\cline { 2 - 5 } \multicolumn{1}{c|}{} & \multicolumn{2}{c|}{$p_{c}$} & \multicolumn{2}{c|}{$k_{\text {sec }}$} \\
\hline & Mean [kN/mm] & CoV & Mean $\left[\mathbf{k N} / \mathbf{m m}^{2}\right]$ & CoV \\
\hline Whole set & 0.225 & 0.382 & 0.345 & 0.205 \\
\hline Reduced set & 0.219 & 0.390 & 0.363 & 0.179 \\
\hline FOSM & 0.210 & 0.512 & 0.378 & 0.174 \\
\hline
\end{tabular}




\section{Conclusions}

In this study, a global sensitivity analysis (GSA) of the behaviour of masonry arch bridges under vertical loading is carried out employing the method of Sobol, allowing the identification of the most influential mechanical parameters. An advanced mesoscale modelling strategy is used to describe the masonry arch bridge behaviour, and a kriging metamodel is employed to replace the computationally expensive finite element models for the purpose of carrying out the numerous simulations required by GSA.

The efficiency of the proposed approach is demonstrated first by considering an analytical example, and by comparing the estimates of the sensitivity indices obtained by using the metamodel and the analytical function. Subsequently, two different case studies are analysed, consisting respectively in a masonry arch for two different values of the rise-to-span ratio, and in a masonry arch bridge. The output parameters of interest are the load capacity, related to the ultimate bridge behaviour, and the secant stiffness, related to the bridge serviceability.

The results of the application of GSA to the masonry arch models show that:

- The parameters that influence the arch load capacity most related to the backfill properties, specifically are the friction angle $\tan \phi_{0}$, followed by the cohesion $c_{0}$ and the parameter $s_{0}$ associated with to the tensile resistance.

- The parameters that control the secant stiffness are the brick Young's modulus $E_{b}$ and the mortar interfaces normal stiffness, $k_{N}$, followed by the parameters that control the mortar resistance.

- The sensitivity to the mechanical parameters of the arch is not significantly affected by the rise-to-span ratio, i.e., the values of the sensitivity indices for the deep arch are very similar to those of the shallow one.

The results of the application of GSA to the masonry arch bridge show that: 
- The load capacity is significantly affected by the cohesion of the backfill, whereas the parameters related to the arch characteristics are less important. Thus, the description of the behaviour of the backfill through an appropriate model and an accurate characterisation of the backfill model parameters and of their uncertainty is of paramount importance for the bridge capacity assessment. Moreover, retrofit strategies aimed at improving the properties of the backfill are expected to lead to a significant increase of the bridge ultimate capacity compared to other techniques aimed at improving the arch response.

- The secant stiffness depends significantly on the parameters influencing the arch stiffness, i.e. the brick young modulus $E_{b}$ and the mortar interfaces normal stiffness, $k_{N}$. The Young's modulus of the soil $E_{f}$ has a lower sensitivity index.

Finally, it has been shown that good estimates of the mean response of interest are obtained by considering only few influential parameters as random, assuming the others as deterministic. A higher number of parameters has to be assumed as uncertain for the purpose of estimating the response dispersion. The first-order-second-moment technique provides reasonably good response estimates for the problems investigated in this study.

The procedure proposed for the sensitivity analysis and uncertain propagation, and the results of the analyses, are relevant to the management of masonry arch bridges, in particular for maintenance activities, such as rehabilitation, strengthening and replacement. Future studies will aim at addressing the sensitivity of the bridge response for other type of loadings such as those induced by floods, requiring the use of more complex three-dimensional bridge models.

\section{Aknowledgements}

The financial support of the European Commission through the Marie Skłodowska-Curie Individual fellowship IF ("FRAMAB", Grant Agreement 657007) for the first author is greatly acknowledged. The authors also acknowledge the Research Computing Service at Imperial College for providing and supporting the required High Performance Computing facilities. 


\section{References}

Andersson, A. (2011). Capacity assessment of arch bridges with backfill: Case of the old Årsta railway bridge (Doctoral Dissertation). KTH Royal Institute of Technology.

Au, S.-K., \& Wang, Y. (2014). Engineering risk assessment with subset simulation, John Wiley $\&$ Sons.

Brencich, A., \& Gambarotta, L. (2005), Mechanical response of solid clay brickwork under eccentric loading. Part I: Unreinforced masonry, Materials and Structures, 38(276), 257266.

Brencich, A., Gambarotta, L., \& Sterpi, E. (2007). Stochastic distribution of compressive strength: effects on the load carrying capacity of masonry arches. Proc. Arch '07 $5^{\text {th }}$ Int. Conf. on Arch Bridges, 641-648.

Brencich, A., \& De Felice, G. (2009). Brickwork under eccentric compression: Experimental results and macroscopic models, Construction and Building Materials, 23(5), 1935-1946.

Casas, J.R. (2011). Reliability-based assessment of masonry arch bridges. Construction and Building Materials, 25(4), 1621-1631.

Cavalagli, N., Gusella, V., \& Severini, L. (2017). The safety of masonry arches with uncertain geometry. Computers and Structures, 188, 17-31.

de Arteaga, I., Morer, P. (2012). The effect of geometry on the structural capacity of masonry arch bridges. Construction and Building Materials, 34, 97-106.

Riveiro, B., Solla, M., de Arteaga, I., Arias, P., \& Morer, P. (2013). A novel approach to evaluate masonry arch stability on the basis of limit analysis theory and non-destructive geometric characterization. Automation in Construction, 31m 140-8.

Chisari, C., Macorini, L., Amadio, C., \& Izzuddin, B. A. (2018). Identification of mesoscale model parameters for brick-masonry. International Journal of Solid and Structures, 146, 224-240 .

Couckuyt, I., Dhaene, T., Demeester, P. (2014). ooDACE toolbox: a flexible object-oriented Kriging implementation. Journal of Machine Learning Research, 15, 3183-3186.

De Felice, G., \& De Santis, S. (2010). Experimental and numerical response of arch bridge historic masonry under eccentric loading, International Journal of Architectural Heritage, 4(2), 115-137.

EN 1996-1-1(2005), Eurocode 6 - Design of Masonry Structures, Part 1-1 General rules for reinforced and unreinforced masonry structures, The British Standard Institution.

Giannini, R., Pagnoni, T., \& Pinto, P.E. (1996) A Risk analysis of a medieval tower before and after strengthening, Structural Safety, 18(2/3), 81-100.

Gilbert, M. (2007). Limit analysis applied to masonry arch bridges: state-of-the-art and recent developments. Proceedings of 5th International Conference on Arch Bridges (ARCH'07), Funchal, Madeira, Portugal.

Hacıefendioğlu, K., Başağa, H.B., \& Banerjee, S. (2017). Probabilistic analysis of historic masonry bridges to random ground motion by Monte Carlo Simulation using Response Surface Method. Construction and Building Materials, 134(1), 199-209.

Heyman, J. (1969). The safety of masonry arches. International Journal of Mechanical Science, $11,363-85$.

Izzuddin, BA (1991). Nonlinear dynamic analysis of framed structures (Doctoral Dissertation). Imperial College, London, UK. 
Jia, G., \& Taflanidis, A. A. (2013). Kriging metamodeling for approximation of high-dimensional wave and surge responses in real-time storm/hurricane risk assessment. Computer Methods in Applied Mechanics and Engineering, 261, 24-38.

Jie, L., Masia, M. J., \& Stewart M. G. (2017). Stochastic spatial modelling of material properties and structural strength of unreinforced masonry in two-way bending. Structure and Infrastructure Engineering, 13(6), 683-695.

Jokhio, G. A. (2012). Mixed dimensional hierarchic partitioned analysis of nonlinear structural systems (Doctoral Dissertation). Department of Civil and Environmental Engineering, Imperial College, London, UK.

Jokhio, G. A., \& Izzuddin, B. A. (2013). Parallelisation of nonlinear structural analysis using dual partition super elements. Advances in Engineering Software, 60, 81-88.

Jokhio, G.A., \& Izzuddin, B. A. (2015). A dual super-element domain decomposition approach for parallel nonlinear finite element analysis. International Journal for Computational Methods in Engineering Science and Mechanics, 16, 188-212.

Li, G., Rabitz, H., Yelvington, P. E., Oluwole, O. O., Bacon, F., Kolb, C. E., \& Schoendorf, J. (2010). Global sensitivity analysis for systems with independent and/or correlated inputs. The Journal of Physical Chemistry A, 114(19), 6022-6032.

Kaushik, H. B., Rai, D. C., \& Jain, S. K. (2007). Stress-strain characteristics of clay brick masonry under uniaxial compression. Journal of materials in Civil Engineering, 19(9), 728739.

Lophaven, S. N., Nielsen, H. B., \& Sondergaard, J. (2002). DACE-A MATLAB Kriging Toolbox. Technical University of Denmark, Lyngby, Denmark.

Lourenço, P. (1996). Computational strategies for masonry structures (Doctoral Dissertation). Technische Universiteit Delft, Netherlands.

Macorini L., \& Izzuddin B.A. (2011). A non-linear interface element for 3D mesoscale analysis of brick-masonry structures, International Journal for Numerical Methods in Engineering, $85,1584-608$.

Melbourne, C., \& Gilbert, M. (1995). The behaviour of multiring brickwork arch bridges. The Structural Engineer. 73(3), 39-47.

Melbourne, C., Wang, J., Tomor, A., Holm, G., Smith, M., Bengtsson, P. E., Bien, J., Kaminski, T., Rawa, P., Casas, J. R., Roca, P., \& Molins, C. (2007) Masonry Arch Bridges Background document D4.7. Sustainable Bridges. Report number: Deliverable D4.7.

Milani, G., \& Benasciutti, D. (2010). Homogenized limit analysis of masonry structures with random input properties: polynomial response surface approximation and Monte Carlo simulations. Structural Engineering and Mechanics, 34(4), 417-447.

Minga, E., Macorini, L., \& Izzuddin, B. A. (2018). Enhanced mesoscale partitioned modelling of heterogeneous masonry structures. International Journal for Numerical Methods in Engineering, 113(13), 1950-1971.

Minga, E., Macorini, L., \& Izzuddin, B. A. (2018). A 3D Mesoscale Damage-Plasticity Approach for Masonry Structures under Cyclic Loading. Meccanica, 53, 1591-1611.

Moreira ,V.N., Fernandes, J., Matos, J.C., \& Oliveira, D.V. (2016). Reliability-based assessment of existing masonry arch railway bridges. Construction and Building Materials 115: 544-554.

Moreira, V. N., Matos, J. C., \& Oliveira, D. V. (2017). Probabilistic-based assessment of a masonry arch bridge considering inferential procedures. Engineering Structures, 134, 61-73. 
Mukherjee, D., Rao, B. N., \& Prasad, A. M. (2011). Global sensitivity analysis of unreinforced masonry structure using high dimensional model representation. Engineering Structures, 33(4), 1316-1325.

Myers, R. H., \& Montgomery, D. C. (1998). Response Surface Methodology: Process and Product Optimization Using Designed Experiments, Wiley, New York.

Ng, K.H., \& Fairfield, C. (2002). Monte Carlo simulation for arch bridge assessment. Construction and Building Materials, 16, 271-80.

Oliveira, D. V., Lourenço, P. B., \& Lemos, C. (2010). Geometric issues and ultimate load capacity of masonry arch bridges from the northwest Iberian Peninsula. Engineering Structures, 32(12), 3955-3965.

Orban, Z. (2007). UIC Project on Assessment, Inspection and Maintenance of Masonry Arch Railway Bridges. Proceedings of ARCH'07: 5th International Conference on Arch Bridges, Multicomp, Lda Publishers, Madeira.

Page, J. (1993). Masonry Arch Bridges - State of the Art Review. HMSO, London.

Patelli, E., Pradlwarter, H.J., \& Schuëller, G.I. (2010). Global sensitivity of structural variability by random sampling. Computer Physics Communications, 181(12), 2072-2081.

Patelli, E., Broggi, M., De Angelis, M., \& Beer, M. (2014). OpenCossan: An efficient open tool for dealing with epistemic and aleatory uncertainties. Proceedings of Second International Conference on Vulnerability and Risk Analysis and Management (ICVRAM) and the Sixth International Symposium on Uncertainty Modeling and Analysis (ISUMA), Liverpool, UK.

Queipo, N., Haftka, R., Shyy, W., Goel, T., Vaidyanathan, R., \& Tucker, P. K. (2005). Surrogate-based analysis and optimization, Progress in Aerospace Sciences, 41(1), 1-28.

Rohmer, J., \& Foerster, E. (2011). Global sensitivity analysis of large-scale numerical landslide models based on Gaussian-Process meta-modeling, Computers \& geosciences, 37(7), 917927.

Sacks, J., Welch ,W.J., Mitchell, T.J., \& Wynn, H.P. (1989). Design and analysis of computer experiments. Statistical science, 409-423.

Saltelli, A., \& Chan, K., Sensitivity Analysis, Wiley Series in Probability and Statistics, 2000.

Sarhosis, V., De Santis, S., \& De Felice, G. (2017). A review of experimental investigations and assessment methods for masonry arch bridges, Structure and Infrastructure Engineering, 12, 1-19.

Schueremans, L., \& Gemert, D.V. (1999). Evaluating the reliability of structural masonry elements using the response surface technique. Proceedings of the 8 th international conference on durability of building materials and components, May 30 - June 3, Vancouver, Canada.

Schueremans, L, \& Van Gemert, D. Probability Density Functions for Masonry Material Parameters-A Way to Go?. In Lourenco, P. B., Roca, P., Modena, C., \& Agrawal, S. (2006), Structural Analysis of Historical Construction, Vol 2 (Set of 3 Volumes): Possibilities of Numerical and Experimental Techniques, 2, 921.

Sobol', I. M. (1976). Uniformly distributed sequences with additional uniformity properties. USSR Computational Mathematics and Mathematical Physics, 16(5), 236-242.

Sobol' I. (1993). Sensitivity estimates for nonlinear mathematical models, Mathematical Modeling \& Computational Experiments, 1, 407-414.

Sykora, M., \& Holicky, M. (2010). Probabilistic model for masonry strength of existing structures. Engineering Mechanics, 17(1), 61-70. 
Tabbakhha, M., \& Modaressi-Farahmand-Razavi, A. (2016). Analyzing the effect of workmanship quality on performance of unreinforced masonry walls through numerical methods. Computers and Structures, 167, 1-14.

Tabbakhha, M., \& Deodatis, G. (2017). Effect of Uncertainty of Tensile Strength of Mortar Joints on the Behavior of Masonry Walls under Lateral. Journal of Structural Engineering, 143(2), 04016166.

Tubaldi, E., Macorini, L., \& Izzuddin, B. A. (2018). Three-dimensional mesoscale modelling of multi-span masonry arch bridges subjected to scour. Engineering Structures, 165, 486500 .

Zampieri, P., Tecchio, G., da Porto, F., \& Modena C., (2015). Limit analysis for transverse seismic capacity of multi-span masonry arch bridges, Bulletin of Earthquake Engineering, 13(5), 1557-1579.

Zanaz, A., Yotte, S., Fouchal, F., \& Chateauneuf, A. (2016). Efficient masonry vault inspection by monte carlo simulations: Case of hidden defect. Case Studies in Structural Engineering, $5,1-12$.

Zhang, Y., Macorini, L., \& Izzuddin, B. A. (2016). Mesoscale partitioned analysis of brickmasonry arches. Engineering Structures, 124, 142-166.

Zhang, Y., Tubaldi, E., Macorini, L., \& Izzuddin, B.A. (2018a). Mesoscale Partitioned Modelling of Masonry Bridges allowing for Arch-Backfill Interaction. Construction and Building Materials, 173, 820-842.

Zhang, Y., Macorini, L., \& Izzuddin, B. A. (2018b). Numerical investigation of arches in brickmasonry bridges, Structure and Infrastructure Engineering, 14(1), 14-32.

Zhu, F., Zhou, Q., Wang, F., \& Yang, X. (2017). Spatial variability and sensitivity analysis on the compressive strength. Construction and Building Materials, 140, 129-138. 